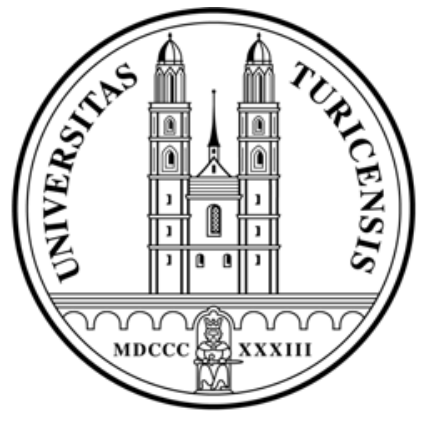

Institute for Empirical Research in Economics

University of Zurich

Working Paper Series

ISSN 1424-0459

Working Paper No. 281

Portfolio Choice with Loss Aversion, Asymmetric Risk-Taking Behavior and Segregation of Riskless Opportunities

Martin Vlcek

April 2006 


\title{
Portfolio Choice with Loss Aversion, Asymmetric Risk-Taking Behavior and Segregation of Riskless Opportunities
}

\author{
Martin Vlcek*
}

May, 12, 2005

April 21, 2006

\begin{abstract}
In this paper we present a two period model, where the agent's preferences are described by prospect theory as proposed by Kahneman and Tversky. We solve for the agent's portfolio decision. Our findings are that the changes in portfolio weights depend crucially on the reference point and the ratio between the reference point and the current wealth, and thus only indirectly on the performance of the risky asset. Our model explains why investor keep on holding, or even buy, loosing investments.
\end{abstract}

Keywords: Disposition effect, house money effect, prospect theory, portfolio choice

\section{Introduction}

*Institute for Empirical Research in Economics, University of Zurich, Blümlisaplstrasse 10, 8006 Zurich, Switzerland. The authors would like to thank for financial support by the national center of competence in research "Financial Valuation and Risk Management". The national centers in research are managed by the Swiss National Science Foundation on behalf of the federal authorities. Email: vlcek@iew.unizh.ch. 
In the finance literature there are conflicting results about the effect of prior outcomes on risk-taking behavior in subsequent periods. Some authors report that investors are prone to the disposition effect, which stands for the tendency to realize gains and to hold loosing investments. The most prominent articles are Shefrin and Statman (1985), Odean (1998) and Weber and Camerer (1998). The standard explication for such a behavior is prospect theory and in particular the fact that investors are risk-seeking in the domain of losses and risk averse in the domain of gains.

However, other studies find the opposite behavior. Thaler and Johnson (1990) observe in their experiments that subjects are risk-seeking after a gain. They term this phenomenon the house-money effect. Further they find evidence for a break-even effect, i.e. that in presence of prior losses, outcomes which offer a chance to break-even are especially attractive. Barberis et al. (2001), who use a linearized value function, present a model, where after prior gains investors become less loss averse while after prior losses they become more loss averse.

In this article we want to shed more light on these issues and present a model where we take asymmetric risk-taking behavior explicitly into account. We consider a two period model for portfolio choice in a stylized financial market where the investor's preferences are described by prospect theory as suggested by Kahneman and Tversky (1979) and Tversky and Kahneman (1992). We investigate the investor's risk-taking behavior following a rise, respectively a fall, in the price of the risky asset.

In our framework, there is a financial market on which two assets are traded. A riskless asset, also called the bond, and a risky asset, the stock. The evolution of the stock prices is described a binomial process.

The investor, who's preferences are based on changes in his wealth and described by prospect theory, chooses a weight in the risky asset to maximize his utility. We assume that he owns an initial endowment and that he earns no other income. Since we want to model a small individual investor, we assume that no short selling is allowed. Further we assume that the investor acts myopically and that the reference point relative to which he measures his gains and losses in the first period is his initial wealth. Following Tversky and Kahneman (1992) we assume a two part power function, reflecting loss aversion and asymmetric risk-taking behavior. Note, that we take the segregation of riskless alternatives during the editing phase explicitly into account.

Our point of interest is the second period behavior of the investor condi- 
tional on the stock price movement in the first period. In particular, we ask if we can explain the behavior of an investor prone to the disposition effect or prone to the house money effect. In our model we define the risk-taking behavior in terms of portfolio weights: an investor who chooses a riskier strategy after a gain and a safer strategy after a loss is a house money investor. If the agent invests less in the risky asset after the stock appreciated and more after it depreciated, we call him a disposition investor. ${ }^{1}$

Whereas in the first period all agents solve the same maximization problem, the second period decision depends on the reference point relative to which the agent measures his second period gains and losses. In our framework there are two prominent candidates for reference points: the initial wealth or the current wealth. We analyze both cases.

For the investor who measures his performance relative to his initial wealth we find the following results: in the first period the agent invests all her wealth in the risk-free bond when it is relatively more attractive than the risky stock. This is the case when the decision weight associated with the good state is lower than the equivalent martingale probability of this state. The more the risky asset gains in attractiveness the more the agent is willing to invest in it. This change occurs gradually. The optimal strategy for a very attractive securities is to invest the whole wealth in the risky asset.

In the second period the portfolio decision looks similar to the first period: if the decision weight for the up state is smaller than the associated equivalent martingale probability, the investor chooses to allocate his entire wealth in the risk-free bond. Note that this condition is the same in both periods and in both states. The more attractive the stock, the higher the fraction invested in it. We observe that after the stock went up in the first period the agent never invests a lower fraction in the risky asset than he did in the first period. If the price of the stock declined in the first period the agent invests the same or a higher fraction of his wealth in the risky asset whenever he experienced a gain in his portfolio. After a loss in wealth, which occurs after the agent chose in the first period to invest his entire wealth in the risky asset, he allocates again the whole wealth in the risky investment opportunity. This case arises only when the stock is very attractive.

Our findings are that the changes in portfolio weights depend crucially on

\footnotetext{
${ }^{1}$ Note that this definition of the disposition effect implies a stronger reaction of the investor to stock price movements than a fix mix strategy. This is consistent with Odean's (1998) results, who showed that investors were reluctant to sell losers even when controlling for rebalancing
} 
the ratio between the reference point and the current wealth, and therefore only indirectly on the performance of the stock.

So even when considering explicitly the asymmetric risk-taking behavior of the investor, a standard explication for the disposition behavior, our investor is not prone to the disposition effect. In particular he chooses a riskier strategy after the stock appreciated. His behavior is essentially determined by loss aversion: a first period gain in wealth cushions possible future losses, so that the investor chooses a riskier strategy.

Further we observe that it is never optimal for the investor to allocate his wealth in a way, that in the next period in both states a loss will occur. That is the investor never puts himself in the loss domain where he is risk-seeking. His loss aversion prevents that he gets that deep into the loss region that in the next period he will be confronted with losses only. If the agent incurs a loss in his portfolio it is because in the first period he invested all his wealth in the risky asset. He does so only when the stock is very attractive, i.e. has a very small loss potential and a very high upside potential. After the bad state realized and the investor incurred a loss in his portfolio, the stock is still very attractive and offers him the possibility to compensate the loss from the previous period. This is the reason why the agent keeps on holding this stock.

Our results are consistent with the house money effect in the sense that investors follow, in relative terms, a riskier strategy after a first period gain. Note that a higher fraction invested in the risky asset, after the stock appreciated, can be consistent with both holding more and less units of the risky asset. So that the results of our model can be consistent, in absolute terms, with a disposition investor's behavior. Our model explains why investor keep on holding, or even, buy loosing investments: either because the stock is very attractive and offers a high chance to undo the loss experienced in the portfolio or either because the gain in the portfolio makes possible future losses more bearable. Empirical tests, taking the relation between stock prices and the portfolio performance into account, have to be conducted to asses the validity of our predictions. An interesting issue is whether the disposition effect arises only in terms of units bought and sold or also in terms of portfolio weights.

The agent who measures his gains and losses relative to his actual wealth solves in each period the same maximizing problem. Therefore he allocates in each period the same fraction of his wealth to the risky asset, that is he chooses a fix mix strategy. An open question remains, if a fix mix strategy 
that implies a rebalancing after each stock price movement can be seen as a cause for the disposition effect.

The rest of the paper is organized as follows. In the next section we describe the framework. In section 3 we present our results and in the last section we offer further discussion of our results and conclude. The appendix contains technical proofs.

\section{The Model}

We present a two period model for portfolio choice in a stylized financial market with two assets where the investor's preferences are described by prospect theory as suggested by Kahneman and Tversky (1979) and Tversky and Kahneman (1992).

In our framework, there is a financial market on which two assets are traded. A riskless asset, also called the bond, and a risky asset, the stock. The evolution of the stock prices is described a binomial process. At the end of the next period there are two possible states. If the stock prices rises, we call the corresponding state the up-state; the other state is called the down state. In the up state, which realizes with probability $p$, the risky investment yields a gross return $R_{U}$. Note that $0<p<1$ (not $\leq$ sonst in $\varphi$ dividieren durch null!). In the down state, arising with probability $1-p$, it yields $R_{D}$. The risk-free bond yields a sure gross return of $R_{f}$. We assume that the time value of money is positive, i.e. that interest rates are non-negative. Absence of arbitrage implies that $R_{U}>R_{f}>R_{D}$. For simplicity and without loss of generality we assume further that $R_{D}<1$. To prevent negative stock prices we assume $R_{D} \geq 0$. These assumptions about the financial market can are summarized in the following statement: $R_{U}>R_{f} \geq 1>R_{D} \geq 0$. All the parameters are assumed to be constant over time.

The preferences of the investor are based on changes in wealth and described by prospect theory. We assume that he owns an initial endowment, $W_{0}$, and that he earns no other income. Since we want to model a small individual investor, we assume that no short selling is allowed. Further we assume that the investor acts myopically ${ }^{2}$ and that the reference point relative to which he measures his gains and losses in the first period is his initial

\footnotetext{
${ }^{2}$ We think that assuming a myopic behavior for a small individual investor is appropriate for a descriptive model.
} 
wealth.

The choice process under prospect theory starts with the editing phase, followed by the evaluation of edited prospects and at the end the alternative with the highest value is chosen.

During the editing phase agents code outcomes into gains and losses and they segregate riskless components in a lottery from its risky components. Moreover they perform additional simplification, we do not consider in our model, such as rounding of probabilities or outcomes. The reason for ignoring the additional simplification, is that the inclusion of theses does not shed more light on our question at hand. In the valuing phase the agents attach a subjective value to the lottery. As proposed by Kahneman and Tversky (1979), the segregation of a riskless component implies that the form of the value function changes, depending on whether there is such a riskless component or not.

In the case without a riskless component, i.e. a lottery involving a gain and a loss, the over all value of a prospect is given by the sum of the subjective values of the outcomes weighted by the agent's decision weights associated with the probability of the outcome. The over all value of a prospect yielding a gain $x$ with probability $p$ and a loss $y$ with probability $1-p$ is given by : $V(x, p ; y, 1-p)=w(p) v(x)+w(1-p) v(y)$; where $x \geq 0 \geq y$. The decision weights $w$ measure the impact of events on the desirability of prospects, and are not merely the perceived likelihood of these events ${ }^{3}$. The function $v$ assigns to each outcome $x$, edited as a gain or a loss, a number $v(x)$ which reflects the subjective value of that outcome. The functional form of $v$ is described bellow.

In the case where there is a riskless component, i.e. where the lottery offers either two gains or two losses, the over all value of the prospect has the following form: $V(x, p ; y, 1-p)=v(y)+w(p)[v(x)-v(y)]$, where $x>y \geq 0$ or $x<y \leq 0$. The fact that, e.g. in the cases of two gains, the agent perceives the lower gain as a sure one ${ }^{4}$ is called segregation. The risky part of the lottery is now the difference between the higher gain and the lower gain occurring with the probability of the higher gain.

As a possible form of the function assigning the subjective value to an outcome Tversky and Kahneman (1992) proposed a two part power function.

\footnotetext{
${ }^{3}$ Following Tversky and Kahneman (1992) we take the following form: $w(p)=$ $\frac{p^{\gamma}}{\left(p^{\gamma}+(1-p)^{\gamma}\right)^{\frac{1}{\gamma}}}$.

${ }^{4}$ Note that an outcome occurring with probability one implies a decision weight of one.
} 
This function describes the experimental evidence the authors found. The key features of their theory are the coding of outcomes into gains and losses, that a loss hurts more than an equivalent gain and asymmetric risk-taking behavior

$$
v(x)=\left\{\begin{array}{ll}
(x)^{\alpha} & \text { if } x \geq 0 \\
-\beta(-x)^{\alpha} & \text { if } x<0
\end{array} .\right.
$$

The function $v$ assigns to each outcome $x$, edited as gain or a loss, a number $v(x)$ which reflects the subjective value of that outcome. $\beta$ is the coefficient of loss aversion and reflects the fact, that losses hurt more than equivalent gains, which is true for all $\beta>1$. Using data from their experiments the authors estimated $\beta$ to equal 2.25. The coefficient $\alpha$ measures the agent's risk aversion and takes on values between zero and one. Using data from their experiments the authors estimated $\alpha$ to equal 0.88. Observe that in the domain of gains, i.e. $x \geq 0$, the value function is concave, implying that the agent is risk averse, whereas for the domain of losses the function is convex, i.e. the investor is risk-seeking in this domain. We assume that all the parameters are constant over time.

\section{Results}

In our two period model for portfolio choice the investor, who's preferences are based on changes in his wealth and described by prospect theory, chooses a weight in the risky asset to maximize his utility. We investigate in particular the investor's behavior after a rise respectively a fall in the price of the risky asset.

\subsection{First Period}

In the first period the investor's portfolio decision consists of allocating his initial wealth to the two assets traded in the financial market. He maximizes his utility in $t=0$ by allocating a fraction $\lambda_{0}$ of his initial wealth in the risky asset and $1-\lambda_{0}$ in the riskless asset. Since we want to model a small individual investor we do not allow for short sales and restrict thus the fraction of wealth invested in the risky asset to be between zero and one. We 


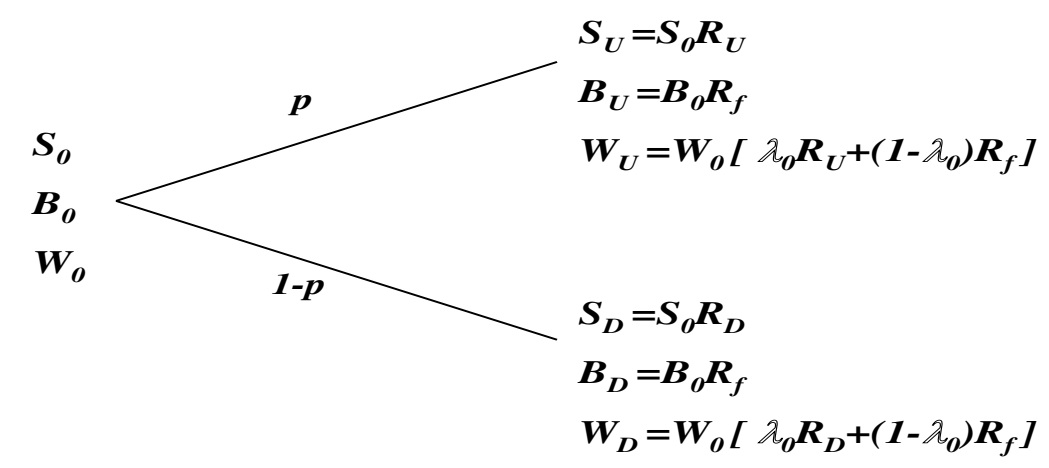

Figure 1: Binomial tree for the first period.

assume that the investor is a myopic optimizer and thus that for his investment decision he takes only the first period into account. The situation he is confronted with at time zero is depicted in figure 1 .

In $t=0$ the stock is worth $S_{0}$, the bond $B_{0}$ and the investor owns his initial wealth $W_{0}$. With probability $p$ the stock prices goes up and the good state realizes. In this case the stock is worth $S_{U}=S_{0} R_{U}$, the bond price is worth $B_{U}=B_{0} R_{f}$ and the investors wealth is $W_{U}$. Note that we skip the time index in $t=1$ and index variables simply by the unambiguous short hander $U$, for the up state in $t=1$, and $D$ for the down state in $t=1$. The investor's wealth position in the up state equals his initial wealth multiplied by the portfolio return, where $\lambda_{0}$ is the fraction of wealth invested in the risky asset. Under the above assumption, and in particular if $\lambda_{0}$ is nonnegative and $R_{U}>R_{f} \geq 1, W_{U}$ is always exceeds the initial wealth, except for the trivial case where the risk-free rate is zero and the agent does not invest in the risky asset. Therefore the investor experiences with every investment strategy a gain in the good state.

With probability $1-p$ the bad state realizes and the stock price depreciates. In this case it is worth $S_{D}=S_{0} R_{D}$. The riskless bond yields the certain gross return of $R_{f}$ and the agent's wealth position is $W_{D}$. Given the setting, the wealth in the down state can be greater, equal or smaller 
than initial position. The performance depends on the returns offered by the traded securities and the portfolio choice of the investor. Taking the return parameters as fixed, the investor's wealth in the bad state is higher for a lower $\lambda_{0}$ and vice versa. More precisely the agent experiences a gain in the bad state when he chooses a $\lambda_{0} \in\left[0, \frac{R_{f}-1}{R_{f}-R_{D}}\right)$. For $\lambda_{0}=\frac{R_{f}-1}{R_{f}-R_{D}}$ he makes neither a gain nor a loss. A $\lambda_{0} \in\left(\frac{R_{f}-1}{R_{f}-R_{D}}, 1\right]$ implies a loss in the down state. Note that $1>\frac{R_{f}-1}{R_{f}-R_{D}} \geq 0$ for any $R_{f} \geq 1>R_{D} \geq 0$. We can interpret $R_{f}-1$ as the riskless gain and $R_{f}-R_{D}$ as the absolute value of the stock's risk premium in the bad state. For illustration simply take the extreme cases where $\lambda_{0}=0$ or $\lambda_{0}=1$. In the case, where $\lambda=0$ and as long as interest are positive the agent makes a sure gain on his portfolio, implying that his wealth is bigger than his initial wealth, even in the bad state. Conversely if he invest all his wealth in the risky asset he will experience in the down state a loss in his wealth.

The relation between the first period wealth and the initial wealth has implications via the segregation of the risk-free components on the form of the objective function in the maximization problem, depending on whether there is such a riskless component or not. The maximization problem consists of choosing the optimal fraction of wealth invested in the risky asset, $\lambda_{0}$, subject to the short selling restriction. We write the maximization problem

$$
\max _{1 \geq \lambda_{0} \geq 0} \quad V\left(\lambda_{0}\right)
$$

where

$$
\begin{gathered}
V= \begin{cases}w(p)\left(W_{U}-W_{0}\right)^{\alpha}-w(1-p) \beta\left(W_{0}-W_{D}\right)^{\alpha} & \text { if } W_{U}>W_{0}>W_{D} \\
\left(W_{D}-W_{0}\right)^{\alpha}+w(p)\left[\left(W_{U}-W_{0}\right)^{\alpha}-\left(W_{D}-W_{0}\right)^{\alpha}\right] & \text { if } W_{U} \geq W_{D}>W_{0}\end{cases} \\
\text { and } W_{U}=W_{0}\left[\lambda_{0} R_{U}+\left(1-\lambda_{0}\right) R_{f}\right] \text { and } W_{D}=W_{0}\left[\lambda_{0} R_{D}+\left(1-\lambda_{0}\right) R_{f}\right] .
\end{gathered}
$$

During the editing phase individuals code outcomes into gains and losses. In the first period they measure their performance relative to their initial wealth. If there are riskless components they segregate such components. In the first case, i.e. in the case without a riskless component, the individual chooses a $\frac{R_{f}-1}{R_{f}-R_{D}} \leq \lambda_{0} \leq 1$, which implies that depending on which state realizes he will experience either a gain or a loss. The other case where there is a riskless component, i.e. where the investor allocates his wealth in a 
way that there are two gains, arises when the investor chooses a $0 \leq \lambda_{0} \leq$ $\frac{R_{f}-1}{R_{f}-R_{D}}$. Following Tversky and Kahneman (1992), we assume a two part power function, reflecting loss aversion and asymmetric risk-taking behavior. Loss aversion is introduced with the coefficient $\beta$, the coefficient $\alpha$ measures the agent's risk aversion. $w(p)$ is the agent's decision weight associated with the probability $p$.

An important observation is that the objective function for the case where $\frac{R_{f}-1}{R_{f}-R_{D}} \leq \lambda_{0} \leq 1$ is convex in the relevant domain, whereas in the case where $0 \leq \lambda_{0} \leq \frac{R_{f}-1}{R_{f}-R_{D}}$ the objective function is concave.

The implications for the maximization problem are that we have to distinguish the two cases, when solving it. In the concave part of the function, we can apply the standard Kuhn Tucker theorem. In the convex part we know from the Weierstrass Theorem the the maximum must lie on the boundary. By plugging back in the objective function, we decide which of the stationary points is the global maximum. The detailed calculation can be found in the appendix. The next proposition summarizes our results for the first period asset allocation.

Proposition 1. Optimal asset allocation in $t=0$ :

1. If it is optimal to choose a $\lambda_{0}^{*}$ implying $W_{D} \geq W_{0}$ and if $w(p) \leq \frac{R_{f}-R_{D}}{R_{U}-R_{D}}$, then $\lambda_{0}^{*}=0$, if $w(p)>\frac{R_{f}-R_{D}}{R_{U}-R_{D}}$ then $\lambda_{0}^{*}=\frac{\left(R_{f}-1\right)\left(1-\kappa^{\frac{1}{\alpha-1}}\right)}{\left(R_{U}-R_{f}\right) \kappa^{\frac{1}{\alpha-1}}-R_{D}+R_{f}}$, where $\kappa \equiv \frac{w(p)\left(R_{U}-R_{f}\right)}{(1-w(p))\left(R_{f}-R_{D}\right)}$.

2. If it is optimal to choose a $\lambda_{0}^{*}$ implying $W_{0} \geq W_{D}$ and if $V\left(\frac{R_{f}-1}{R_{f}-R_{D}}\right)>$ $V(1)$, then $\lambda_{0}^{*}=\frac{R_{f}-1}{R_{f}-R_{D}}$, else $\lambda_{0}^{*}=1$.

If it is optimal to choose a $\lambda_{0}^{*}$ such that in $t=1$ two gains arise and if $w(p) \leq \frac{R_{f}-R_{D}}{R_{U}-R_{D}}$, then it is optimal to invest the whole wealth in the risk-free asset. Note that in absence of arbitrage and for positive interests $\frac{R_{f}-R_{D}}{R_{U}-R_{D}}$ is grater than zero and smaller than one. We can interpret this term as the ratio of the difference in returns between the riskless asset and the stock's return in the bad state and the spread between the stock's possible returns. The higher the stock return in the good state, ceteris paribus, the lower the chance that $\lambda_{0}^{*}$ equals zero. The higher the risk-free rate, the higher the chance that $\lambda_{0}^{*}$ equals zero. Further $\frac{R_{f}-R_{D}}{R_{U}-R_{D}}$ is the martingale probability of the up state. 
So whenever the investor's decision weight associated with the probability of the up state is smaller than the martingale probability of that state, he will invest all his wealth in the risk-free asset. If the decision weight, i.e. the impact of the outcome of the good state, exceeds the martingale probability he will invest the fraction $\frac{\left(R_{f}-1\right)\left(1-\kappa^{\frac{1}{\alpha-1}}\right)}{\left(R_{U}-R_{f}\right) \kappa^{\frac{1}{\alpha-1}}-R_{D}+R_{f}}$ in the risky asset, where $\kappa$ is the ratio of the risk premia weighted by the corresponding decision weights. Note that this decision is independent of the loss aversion coefficient $\beta$. In the case where it is optimal to choose a high $\lambda_{0}$ the agent choose one of the boundaries, i.e. either $\frac{R_{f}-1}{R_{f}-R_{D}}$ or 1 , depending on which of the both choices offers the higher utility. Note that the optimal investment strategy is independent of the initial wealth of the investor.

Next let's turn to the question, when it is optimal to allocate the wealth in a way that two gains arise in $t=1$ and when such that a gain and a loss may arise. In what follows the expression $V_{G G}$ stands for the value function in the case where two gains arise, i.e. the case where the agent segregates the riskless component. $V_{G L}$ stands for the value function when no segregation takes place. The agent will choose a $\lambda_{0}$ such that there are two gains, i.e. $0 \leq \lambda_{0} \leq \frac{R_{f}-1}{R_{f}-R_{D}}$, whenever $V_{G G}\left(0 \leq \lambda_{0} \leq \frac{R_{f}-1}{R_{f}-R_{D}}\right) \geq V_{G L}\left(1 \geq \lambda_{0}>\frac{R_{f}-1}{R_{f}-R_{D}}\right)$ or equivalently if

$$
\begin{array}{r}
V_{G G}\left(0<\lambda_{0}<\frac{R_{f}-1}{R_{f}-R_{D}}\right) \geq V_{G L}\left(\lambda_{0}=\frac{R_{f}-1}{R_{f}-R_{D}}\right) \\
\left(\frac{1}{\left(R_{U}-R_{f}\right) \kappa^{\frac{1}{\alpha-1}}+R_{f}-R_{D}}\right)^{\alpha}\left((1-w(p)) \kappa^{\frac{\alpha}{\alpha-1}}+w(p)\right) \geq w(p)\left(\frac{1}{R_{f}-R_{D}}\right)^{\alpha}
\end{array}
$$

and

$$
\begin{array}{r}
V_{G G}\left(0<\lambda_{0}<\frac{R_{f}-1}{R_{f}-R_{D}}\right) \geq V_{G L}\left(\lambda_{0}=1\right) \\
(1-w(p))\left(\left(R_{D}-R_{f}\right) \frac{\left(R_{f}-1\right)\left(1-\kappa^{\frac{1}{\alpha-1}}\right)}{\left(R_{U}-R_{f}\right) \kappa^{\frac{1}{\alpha-1}}-\left(R_{D}-R_{f}\right)}+R_{f}-1\right)^{\alpha} \\
+w(p)\left(\left(R_{U}-R_{f}\right) \frac{\left(R_{f}-1\right)\left(1-\kappa^{\frac{1}{\alpha-1}}\right)}{\left(R_{U}-R_{f}\right) \kappa^{\frac{1}{\alpha-1}}-\left(R_{D}-R_{f}\right)}+R_{f}-1\right)^{\alpha} \\
\geq w(p)\left(R_{U}-1\right)^{\alpha}-w(1-p) \beta\left(1-R_{D}\right)^{\alpha}
\end{array}
$$


or

$$
\begin{array}{r}
V_{G G}\left(\lambda_{0}=0\right) \geq V_{G L}\left(\lambda_{0}=\frac{R_{f}-1}{R_{f}-R_{D}}\right) \\
w(p) \leq\left(\frac{R_{f}-R_{D}}{R_{U}-R_{D}}\right)^{\alpha}
\end{array}
$$

and

$$
\begin{array}{r}
V_{G G}\left(\lambda_{0}=0\right) \geq V_{G L}\left(\lambda_{0}=1\right) \\
\left(R_{f}-1\right)^{\alpha} \geq w(p)\left(R_{U}-1\right)^{\alpha}-w(1-p) \beta\left(1-R_{D}\right)^{\alpha} .
\end{array}
$$

This inequalities give the conditions the parameters of the problem have to satisfy for the agent to prefer two possible gains over a possible gain and a possible loss, that is to prefer a bearish strategy over a bullish one. The condition involves the parameters of the agent's preferences, $\alpha, \beta$ and $w(p)$, as well as the parameters of the financial market, i.e. the possible returns and the probabilities for the possible states. Since many parameters parameters are involved it is not possible to find closed form solutions for this inequalities of higher order. Therefore we present numerical results for the optimal allocation of wealth in $t=0$.

Figure 2 shows the optimal $\lambda_{0}^{*}$ s for different stock returns $R_{U}$ and $R_{D}$. $R_{U}$ ranges from 1 to 2 and $R_{D}$ from 0 to 1 . The risk-free rate is fixed at 0.1 , $\alpha=0.88, \beta=2.25$ and $\gamma=0.65$. The probability of the good state equals 0.5 .

In the corner, where the return of the stock in the good state is very close to the risk-free return and where the gross return in the down state is close to zero, $\lambda_{0}^{*}=0$, since in this case the risk-free investment is more attractive than the risky stock. When the risky assets offers more attractive returns the agent invest gradually more in the stock. When the stock is very attractive, the investor chooses to allocate his entire wealth in the risky asset.

\subsection{Second Period}

As we assume that in our model all the parameters are constant over time, the setting in the second period has the same structure as in the first period. 


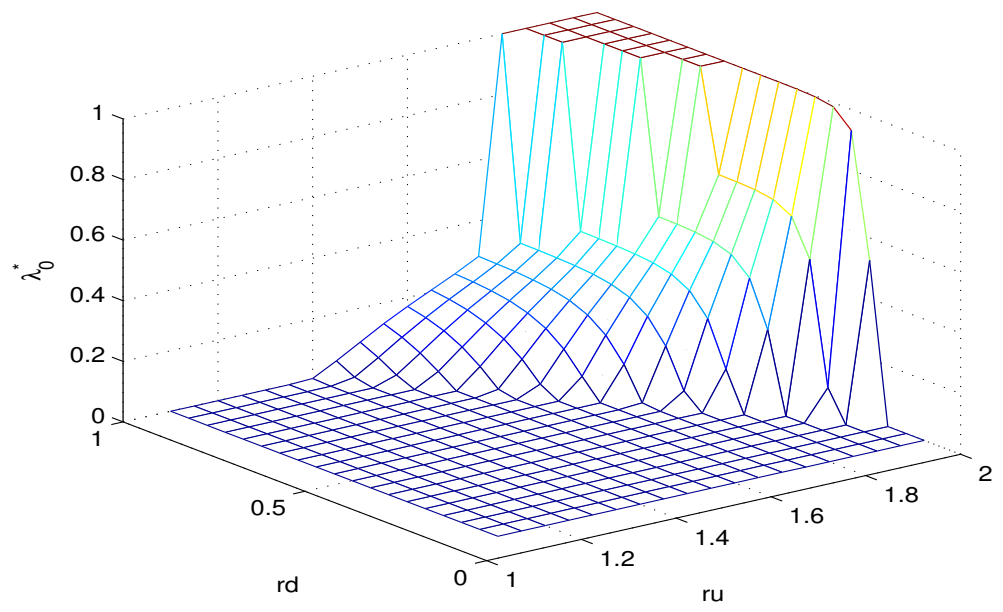

Figure 2: $\lambda_{0}^{*}$ for different stock returns $R_{U}$ and $R_{D}$. The risk-free rate is fixed at $0.1, p=0.5, \alpha=0.88, \beta=2.25$ and $\gamma=0.65$.

After the investor has made his first period investment decision the state of nature in $t=1$ realizes. The market parameters, the investment decision $\lambda_{0}$ and the realized state of nature determine the agent's wealth in $t=1$. In the second period the investor allocates his wealth in $t=1$ to the two assets traded in the financial market to maximize his utility. The situation he is confronted with is shown in figure 3.

We will continue to skip time indices and to label the nodes of the binomial tree with the shorthanders $0, U, D, U U, U D, D U$, and $D D$ where $0, U, D$ are as in the first period, $U U$ stands for the node after two up movements, $U D$ for an up movement followed by a down movement, $D U$ for a down movement followed by an up movement and $D D$ for two consecutive down movements. In the same sense we will call $\lambda_{0}$ the fraction of wealth invested in the risky asset in $t=0, \lambda_{U}$ is the fraction of wealth invested in the risky asset in $t=1$, given the stock went up in the first period and $\lambda_{D}$ is the fraction of wealth invested in the risky asset in $t=1$, given the stock went down in the first period. The asset prices in $t=2$ are standard. The investors wealth position in $t=2$ equals his position in $t=1$ multiplied by the return of his portfolio in the second period. Note that the wealth position at the end of the second period depends on the first and second period decision of the investor. 


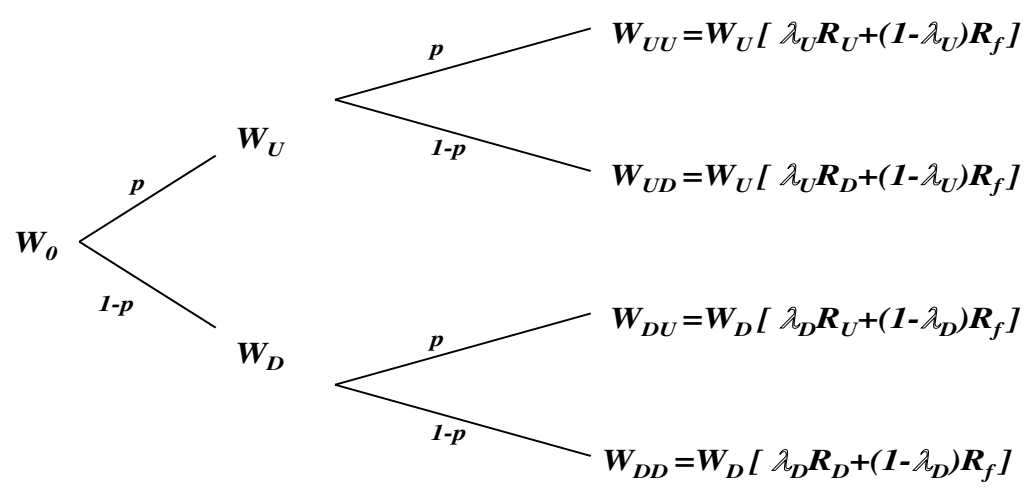

Figure 3: Binomial tree for two periods.

Whereas in the first period all agents solve the same maximization problem, the second period decision depends on an additional characteristic of the investor. It is the reference point relative to which the agent measures his gains and losses ${ }^{5}$. In our model there are two prominent candidates for reference points: the initial wealth $W_{0}$ or the current wealth $W_{U}$, respectively $W_{D}$.

An agent who measures his gains and losses relative to his current wealth treats each gain and loss separately: e.g. let $W_{0}=100, W_{1}=90$ and $W_{2}=80$, in this case the investor perceives the two-period outcome as two consecutive losses each of the magnitude of 10 . The same applies for two consecutive gains. A relatively small loss after a large gain is valued as a large gain and a separate loss, where the overall value of both outcomes depends on the agents loss aversion and risk attitudes; similarly for a loss followed a gain.

An investor, who uses the initial wealth as his reference point implicitly adds up all losses and gains, that is he nets his positions. E.g. if he has a initial wealth position of $W_{0}=100$ and his wealth rises in the next two periods, say $W_{1}=110$ and $W_{2}=120$, he values his position at $t=2$ as a

\footnotetext{
${ }^{5}$ Note that the reference point is a characteristic of the individual, not a choice variable.
} 
gain of 20 and not as two gains of 10. This distinction matters, since the value function is assumed to be concave in the domain of gains and convex in the domain of losses. The same argument applies for two consecutive losses. A relatively small loss after a large gain is perceived as a gain; a large loss after a small gain as a loss and so on.

First we analyze the portfolio choice of the investor who's reference point is his current wealth. Then we solve the maximization problem of the agent who measures his performance relative to his initial wealth. The optimal decision rule for the investor who's reference point is his current wealth is given in proposition 2 .

Proposition 2. The agent who measures his gains and losses relative to his current wealth solves in each period the same maximizing problem. Therefore he allocates in each period the same fraction of his wealth to the risky asset, that is $\lambda_{0}^{*}=\lambda_{U}^{*}=\lambda_{D}^{*}$.

We observe that the investor who measures his gains and losses relative to his current wealth plays a fix-mix strategy. This is not that surprising, since he does not consider the past performance of his portfolio in his current investment decisions.

Next we analyze the behavior of the investor who measures his performance relative to his initial wealth. We start with the decision after the good state realized in the first period. Then we will analyze his behavior after the stock price declined.

Given that in the first period the good state realized, with probability $p$ the state $U U$ realizes. Under the above assumptions and in particular if $\lambda_{U} \geq 0$ and $R_{U}>R_{f}>1$ the wealth position in this state exceeds the initial wealth, i.e. $W_{U U}>W_{0}$, for any strategy. Therefore the investor experiences with every investment strategy a gain in the state $U U$.

With probability $1-p$ state $U D$ realizes implying that the investors wealth position is $W_{U D}$. Given the setting, the wealth in this state can be smaller, equal or greater than the investors's reference point. Taking the return parameters as fixed, the investor's wealth is higher for lower $\lambda_{U}$ and vice versa. More precisely, the investor experiences a gain if he chooses a $\lambda_{U} \in\left[0, \frac{R_{f}-\frac{W_{0}}{W_{U}^{*}}}{R_{f}-R_{D}}\right)$. In the case where $\lambda_{U}=\frac{R_{f}-\frac{W_{0}}{W_{U}^{*}}}{R_{f}-R_{D}}$ he makes neither a gain, nor a loss. A $\lambda_{U} \in\left(\frac{R_{f}-\frac{W_{0}}{W_{U}^{*}}}{R_{f}-R_{D}}, 1\right]$ implies a loss. Note that the boundary $\frac{R_{f}-\frac{W_{0}}{W_{U}^{*}}}{R_{f}-R_{D}}$ is always positive. It is smaller than one whenever $\frac{W_{0}}{W_{U}}>R_{D}$, which 
arises when $\lambda_{0}^{*}<\frac{1-R_{f} R_{D}}{R_{D}\left(R_{U}-R_{f}\right)}$. Note that $\lambda_{0}^{*}$ in turn has to be positive, which is the case when $R_{f} R_{D} \leq 1$. Further note that $W_{U}^{*}$ is always greater or equal than the initial wealth.

The relation between the second period wealth and the initial wealth has implications via the segregation of the risk-free components on the form of the objective function in the maximization problem, depending on whether there is such a riskless component or not. The maximization problem consists of choosing the optimal fraction of wealth invested in the risky asset, $\lambda_{U}$, subject to the short selling restriction. We write the maximization problem

$$
\max _{1 \geq \lambda_{U} \geq 0} \quad V\left(\lambda_{U}\right)
$$

where

$$
\begin{gathered}
V=\left\{\begin{array}{l}
V_{U G L} \text { if } W_{U U}>W_{0}>W_{U D} \\
V_{U G G} \text { if } W_{U U} \geq W_{U D}>W_{0}
\end{array}\right. \\
V_{U G L}=w(p)\left(W_{U U}-W_{0}\right)^{\alpha}-w(1-p) \beta\left(W_{0}-W_{U D}\right)^{\alpha}, \\
V_{U G G}=\left(W_{U D}-W_{0}\right)^{\alpha}+w(p)\left[\left(W_{U U}-W_{0}\right)^{\alpha}-\left(W_{U D}-W_{0}\right)^{\alpha}\right] \\
W_{U U}=W_{U}^{*}\left[\lambda_{U} R_{U}+\left(1-\lambda_{U}\right) R_{f}\right], W_{U D}=W_{U}^{*}\left[\lambda_{U} R_{D}+\left(1-\lambda_{U}\right) R_{f}\right], \\
\text { and } W_{U}^{*}=W_{0}\left[\lambda_{0}^{*} R_{U}+\left(1-\lambda_{0}^{*}\right) R_{f}\right] .
\end{gathered}
$$

During the editing phase individuals code outcomes into gains and losses. They measure their performance relative to their initial wealth. If there are riskless components they segregate such components. In the first case, i.e. in the case without a riskless component, the individual chooses a $\frac{R_{f}-\frac{W_{0}}{W_{U}^{*}}}{R_{f}-R_{D}} \leq \lambda_{U} \leq 1$, which implies that depending on which state realizes he will experience either a gain or a loss. The other case where there is a riskless component, i.e. where the investor allocates his wealth in a way that in both states a gain arises, follows when the investor chooses a $0 \leq \lambda_{U} \leq \frac{R_{f}-\frac{W_{0}}{W_{U}^{*}}}{R_{f}-R_{D}}$. We assume a two part power function, reflecting loss aversion and asymmetric risk-taking behavior. Loss aversion is introduced with the coefficient $\beta$, the coefficient $\alpha$ measures the agent's risk aversion. $w(p)$ is the agent's decision weight associated with the probability $p$.

An important observation is that the objective function for the case where $\frac{R_{f}-\frac{W_{0}}{W_{U}^{*}}}{R_{f}-R_{D}} \leq \lambda_{U} \leq 1$ is convex in the relevant domain, whereas in the case where $0 \leq \lambda_{U} \leq \frac{R_{f}-\frac{W_{0}}{W_{U}^{*}}}{R_{f}-R_{D}}$ the objective function is concave. 
The implications for the maximization problem are that we have to distinguish the two cases, when solving it. In the concave part of the function, we can apply the standard Kuhn Tucker theorem. In the convex part we know from the Weierstrass Theorem the the maximum must lie on the boundary. By plugging back in the objective function, we decide which of the stationary points is the global maximum. The detailed calculation can be found in the appendix. The next proposition summarizes our results.

Proposition 3. Optimal asset allocation in $t=U$ of the investor who measures his performance relative to his initial wealth:

1. If it is optimal to choose a $\lambda_{U}^{*}$ implying a gain in both states, i.e. $W_{U U} \geq$ $W_{U D} \geq W_{0}$ and if $w(p) \leq \frac{R_{f}-R_{D}}{R_{U}-R_{D}}$, then $\lambda_{U}^{*}=0$. If $w(p)>\frac{R_{f}-R_{D}}{R_{U}-R_{D}}$, then $\lambda_{U}^{*}=\frac{\left(R_{f}-\frac{W_{0}}{W_{U}}\right)\left(1-\kappa^{\frac{1}{\alpha-1}}\right)}{\left(R_{U}-R_{f}\right) \kappa^{\frac{1}{\alpha-1}}-R_{D}+R_{f}}$, where $\kappa \equiv \frac{w(p)\left(R_{U}-R_{f}\right)}{(1-w(p))\left(R_{f}-R_{D}\right)}$. If further $\frac{R_{f}-\frac{W_{0}}{W_{U}^{*}}}{R_{f}-R_{D}} \geq 1$ and $\kappa^{\frac{1}{\alpha-1}} \leq \frac{R_{D}-\frac{W_{0}}{W_{U}^{*}}}{R_{U}-\frac{W_{0}}{W_{U}^{*}}}$ then $\lambda_{U}^{*}=1$.

2. If it is optimal to choose a $\lambda_{U}^{*}$ implying a gain in the up state and a loss in the down state, i.e. $W_{U U}>W_{0} \geq W_{U D}$ and if $V\left(\frac{R_{f}-\frac{W_{0}}{W_{U}^{*}}}{R_{f}-R_{D}}\right)>V(1)$, then $\lambda_{U}^{*}=\frac{R_{f}-\frac{W_{0}}{W_{U}^{*}}}{R_{f}-R_{D}}$, else $\lambda_{U}^{*}=1$.

Note that, the condition for $\lambda_{U}^{*}$ to be zero is the same as for $\lambda_{0}^{*}$, namely that the decision weight associated with the good state has to be smaller than the equivalent martingale probability of this state. If this condition is not satisfied and still it is optimal to allocate such that two gains arise then $\lambda_{U}^{*}=\frac{\left(R_{f}-\frac{W_{0}}{W_{U}}\right)\left(1-\kappa^{\frac{1}{\alpha-1}}\right)}{\left(R_{U}-R_{f}\right) \kappa^{\frac{1}{\alpha-1}}-R_{D}+R_{f}}$. Compared with the solution in $t=0$ we see that the first term in the numerator changes. It is the difference between the gross risk-free return and the ratio between the reference point and the current wealth, which in $t=0$ equals one. ${ }^{6}$

If the condition for $\lambda_{U}^{*}=0$ is not satisfied and further $\frac{R_{f}-\frac{W_{0}}{W_{U}^{*}}}{R_{f}-R_{D}} \geq 1$ and $\kappa^{\frac{1}{\alpha-1}} \leq \frac{R_{D}-\frac{W_{0}}{W_{U}^{*}}}{R_{U}-\frac{W_{0}}{W_{U}^{*}}}$ then $\lambda_{U}^{*}=1$. The condition $\frac{R_{f}-\frac{W_{0}}{W_{U}^{*}}}{R_{f}-R_{D}} \geq 1$ implies that the

\footnotetext{
${ }^{6}$ The same observation applies to the case where $\lambda_{U}^{*}=\frac{R_{f}-\frac{W_{0}}{W_{U}^{*}}}{R_{f}-R_{D}}$.
} 
boundary for $\lambda_{U}$ implying two gains is bigger than one, where as from $\kappa^{\frac{1}{\alpha-1}} \leq$ $\frac{R_{D}-\frac{W_{0}}{W_{U}^{*}}}{R_{U}-\frac{W_{0}}{W_{U}^{*}}}$ it follows that $\frac{\left(R_{f}-\frac{W_{0}}{W_{U}^{*}}\right)\left(1-\kappa^{\frac{1}{\alpha-1}}\right)}{\left(R_{U}-R_{f}\right) \kappa^{\frac{1}{\alpha-1}}-R_{D}+R_{f}} \geq 1$ so that the optimal allocation is bounded by one, i.e. $\lambda_{U}^{*}=1$. In the case where it is optimal to choose a high $\lambda_{U}$ the agent choose one of the boundaries, i.e. either $\frac{R_{f}-\frac{W_{0}}{W_{U}^{*}}}{R_{f}-R_{D}}$ or 1 , depending on which of the both choices offers the higher utility. Note that for $\lambda_{U}^{*}$ implying either a gain or a loss in $t=2$, i.e. $W_{U U}>W_{0} \geq W_{U D}$ we need the boundary between the allocation implying two gains and the one implying a gain and al loss to be smaller than one, i.e. $\frac{R_{f}-\frac{W_{0}}{W_{U}^{*}}}{R_{f}-R_{D}} \leq 1$. Otherwise the first case, i.e. where $\lambda_{U}^{*}$ such that $W_{U U} \geq W_{U D} \geq W_{0}$ applies.

From this we see that the factor which alters the allocation between $t=0$ and $t=U$ is the ratio between the reference point, i.e. the initial wealth, and the current wealth ${ }^{7}$. Since after a rise in stock prices the first period wealth never is smaller than the initial wealth, a higher or the same fraction of wealth is invested in the risky asset.

Next let's turn to the question, when it is optimal to allocate the wealth in a way that two gains arise in $t=2$ and when such that a gain and a loss may arise. In what follows the expression $V_{G G}$ stands for the value function in the case where two gains arise, i.e. the case where the agent segregates the riskless component. $V_{G L}$ stands for the value function when no segregation takes place. The agent will choose a $\lambda_{U}$ such that there are two gains, i.e. $0 \leq \lambda_{U} \leq \frac{R_{f}-\frac{W_{0}}{W_{U}^{*}}}{R_{f}-R_{D}}$, whenever $V_{G G}\left(0 \leq \lambda_{U} \leq \frac{R_{f}-\frac{W_{0}}{W_{U}^{*}}}{R_{f}-R_{D}}\right) \geq V_{G L}\left(1 \geq \lambda_{U}>\right.$ $\left.\frac{R_{f}-\frac{W_{0}}{W_{U}^{*}}}{R_{f}-R_{D}}\right)$ or equivalently if

$V_{G G}\left(0<\lambda_{U G G}<\frac{R_{f}-\frac{W_{0}}{W_{U}^{*}}}{R_{f}-R_{D}}\right) \geq V_{G L}\left(\lambda_{U}=\frac{R_{f}-\frac{W_{0}}{W_{U}^{*}}}{R_{f}-R_{D}}\right)$
$\left(\frac{1}{\left(R_{U}-R_{f}\right) \kappa^{\frac{1}{\alpha-1}}+R_{f}-R_{D}}\right)^{\alpha}\left((1-w(p)) \kappa^{\frac{\alpha}{\alpha-1}}+w(p)\right) \geq w(p)\left(\frac{1}{R_{f}-R_{D}}\right)^{\alpha}$

\footnotetext{
${ }^{7}$ This is the reason for which the investor who's reference point is his current wealth chooses in each period the same allocation, i.e. plays a fix-mix strategy.
} 
and

$$
\begin{array}{r}
V_{G G}\left(0<\lambda_{U G G}<\frac{R_{f}-\frac{W_{0}}{W_{U}^{*}}}{R_{f}-R_{D}}\right) \geq V_{G L}\left(\lambda_{U}=1\right) \\
\left((1-w(p)) \kappa^{\frac{\alpha}{\alpha-1}}+w(p)\right)\left(\frac{\left(R_{f}-\frac{W_{0}}{W_{U}^{*}}\right)\left(R_{U}-R_{D}\right)}{\left(R_{U}-R_{f}\right) \kappa^{\frac{1}{\alpha-1}}+R_{f}-R_{D}}\right)^{\alpha} \\
\geq w(p)\left(R_{U}-\frac{W_{0}}{W_{U}^{*}}\right)^{\alpha}-w(1-p) \beta\left(\frac{W_{0}}{W_{U}^{*}}-R_{D}\right)^{\alpha}
\end{array}
$$

or

$$
\begin{array}{r}
V_{G G}(0) \geq V_{G L}\left(\lambda_{U}=\frac{R_{f}-\frac{W_{0}}{W_{U}^{*}}}{R_{f}-R_{D}}\right) \\
w(p) \leq\left(\frac{R_{f}-R_{D}}{R_{U}-R_{D}}\right)^{\alpha}
\end{array}
$$

and

$$
\begin{array}{r}
V_{G G}(0) \geq V_{G L}\left(\lambda_{U}=1\right) \\
\left(R_{f}-\frac{W_{0}}{W_{U}^{*}}\right)^{\alpha} \geq w(p)\left(R_{U}-\frac{W_{0}}{W_{U}^{*}}\right)^{\alpha}-w(1-p) \beta\left(\frac{W_{0}}{W_{U}^{*}}-R_{D}\right)^{\alpha} .
\end{array}
$$

This inequalities give the conditions the parameters of the problem have to satisfy for the agent to prefer two possible gains over a possible gain and a possible loss, that is to prefer a bearish strategy over a bullish one. The conditions involve the parameters of the agent's preferences, $\alpha, \beta$ and $w(p)$, as well as the parameters of the financial market, i.e. the possible returns and the probabilities for the possible states. Further it has to be consistent with the investor's first period decision. Since so many parameters are involved it is not possible to find closed form solutions for this inequalities of higher order. Therefore we present numerical results for the optimal allocation of wealth in $t=U$.

Note that in the case where $\frac{R_{f}-\frac{W_{0}}{W_{U}^{*}}}{R_{f}-R_{D}} \geq 1$ there exist only one stationary point and therefore we do not have to make this comparisons.

Figure 4 shows the optimal $\lambda_{0}^{*}$ and optimal $\lambda_{U}^{*}$ for different stock returns $R_{U}$ and $R_{D}$. $R_{U}$ ranges from 1 to 2 and $R_{D}$ from 0 to 1 . The risk-free rate is fixed at $0.1, \alpha=0.88, \beta=2.25$ and $\gamma=0.65$. The probability of the good state equals 0.5 . The results for the second period are consistent with optimal first period behavior. 

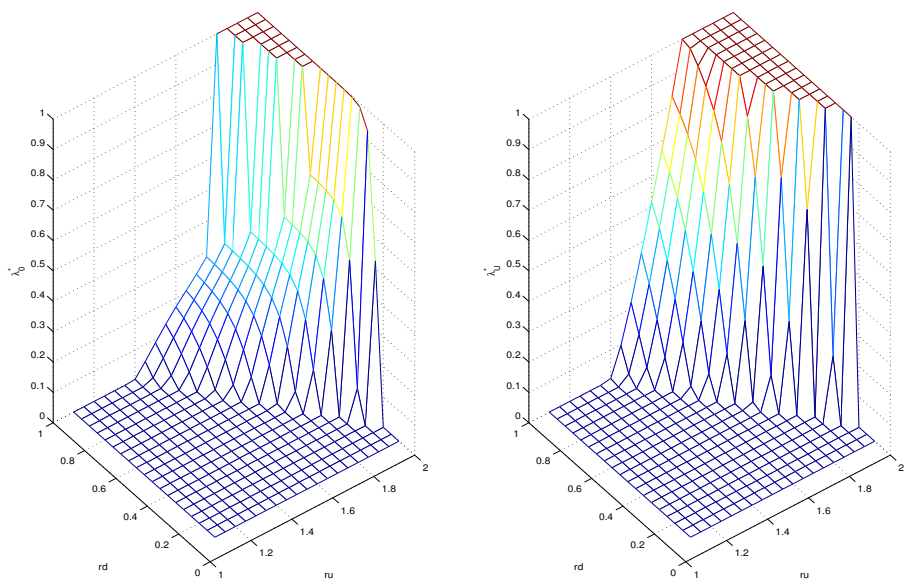

Figure 4: $\lambda_{0}^{*}$ and $\lambda_{U}^{*}$ for different stock returns $R_{U}$ and $R_{D}$. The risk-free rate is fixed at $0.1, p=0.5, \alpha=0.88, \beta=2.25$ and $\gamma=0.65$.

In the corner, where the return of the stock in the good state is very close to the risk-free return and where the gross return in the down state is close to zero, $\lambda_{U}^{*}=0$, since in this case the risk-free investment is more attractive. When the risky assets offers more attractive returns the agent invest gradually more in the stock. When the stock is very attractive, the investor chooses to allocate his entire wealth in the risky asset. Note that the investor chooses to allocate his entire wealth in the risk-free asset for the same characteristics of the two securities. When the risky asset is relatively more attractive than the risk-free one the agent invests more in the stock in $t=1$ than in $t=0$, i.e. $\lambda_{U}^{*}$ goes up steeper than $\lambda_{0}^{*}$. Moreover in $t=U$ the agent invests his entire wealth in the risky asset even if the risky asset offers returns for which the investor in $t=0$ chooses to allocate a fraction smaller than one in it.

Let's turn to the case where the stock went down in the first period and call this state $t=D$. We call the agent's choice variable $\lambda_{D}$. Note that depending on his first period choice the investor experiences after a decline in the stock price in the first period either a gain, a zero gain or a loss. If he chooses a $0 \leq \lambda_{0}^{*}<\frac{R_{f}-1}{R_{f}-R_{D}}$ then his wealth in the state $D$ exceeds his initial wealth, if he invests a fraction $\lambda_{0}^{*}=\frac{R_{f}-1}{R_{f}-R_{D}}$ in the risky asset then he makes 
a zero gain and if he chooses a high $\lambda_{0}^{*}$ he makes a loss on his portfolio. His performance in $t=2$ depends hence on his asset allocation in $t=1$.

If in the first period the agent invested his entire wealth in the risky asset and thus experiences a loss in $t=1$, then in $t=2$ if the state $\mathrm{t}=\mathrm{DU}$ realizes he experiences a loss if he chooses a $\lambda_{D}<\frac{\frac{W_{0}}{W_{D}^{*}}-R_{f}}{R_{U}-R_{f}}$ and if $\frac{W_{0}}{W_{D}^{*}}>R_{f} \geq 1$. That is a low $\lambda_{D}$ cannot undo the first period loss, since the risk-free rate is too low. Note that this case is independent of the relation between $\frac{W_{0}}{W_{D}^{*}}$ and $R_{U}$. The investor makes neither a gain nor a loss if he allocates $\lambda_{D}=\frac{\frac{W_{0}}{W_{D}^{*}}-R_{f}}{R_{U}-R_{f}}$ to the risky asset. The agent makes a gain if he invests $\lambda_{D}>\frac{\frac{W_{0}}{W_{D}^{*}}-R_{f}}{R_{U}-R_{f}}$ and if $R_{U}>\frac{W_{0}}{W_{D}^{*}}>R_{f}>1$. In this case the agent can undo with a high $\lambda_{D}$ the fist period loss if $R_{U}$ is high enough or if the first period loss is small. Note that the condition $\frac{W_{0}}{W_{D}^{*}}>R_{U}$ yields a contradiction with the requirement that $\lambda_{D}$ has to be between zero and one. This means, that the first period loss is to big to be offset by $R_{U}$. If $R_{U}>R_{f}>\frac{W_{0}}{W_{D}^{*}}>1$ then $\frac{\frac{W_{0}}{W_{D}^{*}}-R_{f}}{R_{U}-R_{f}}<0$ so that for all $\lambda_{D}$ a gain results in the good state in $t=2$. The second period wealth positions in the state $D D$ are the following. The investor incurs a loss if he allocates $\lambda_{D}>\frac{R_{f}-\frac{W_{0}}{W_{D}^{*}}}{R_{f}-R_{D}}$, for all $R_{f}, \frac{W_{0}}{W_{D}^{*}}>1$ and $R_{D}<1$. After a first period loss, choosing a high $\lambda_{D}$ results always in a loss in the bad state in $\mathrm{t}=2$. Note that if $\frac{W_{0}}{W_{D}^{*}}>R_{f}$ the boundary for $\lambda_{D}$ is zero. The agent makes a zero gain if his strategy in $t=1$ is $\lambda_{D}=\frac{R_{f}-\frac{W_{0}}{W_{D}^{*}}}{R_{f}-R_{D}}$. He makes a gain if $\lambda_{D}<\frac{R_{f}-\frac{W_{0}}{W_{D}^{*}}}{R_{f}-R_{D}}$ and if $R_{f}>\frac{W_{0}}{W_{D}^{*}}>1>R_{D}$, that is, in the case of a relatively high risk-free rate a low $\lambda_{D}$ generates a gain in $t=D D$. In the opposite case, where $R_{f}<\frac{W_{0}}{W_{D}^{*}}$ there is no possibility to undo the first period loss, even when investing only in the safe asset.

If in the first period it was optimal for the investor to allocate his wealth in a way such that he makes neither a gain nor a loss, i.e. he chose $\lambda_{0}^{*}=\frac{R_{f}-1}{R_{f}-R_{D}}$, then the decision problem in the down state is the same as in $\mathrm{t}=0$.

If the investor makes a gain in the first, i.e. if he chose a $0 \leq \lambda_{0}^{*}<\frac{R_{f}-1}{R_{f}-R_{D}}$, then at the end of the second period in the up state $D U$ there results a gain for all possible investment strategies. In the down state $D D$ the agent experiences a loss if he allocates a fraction $\lambda_{D}>\frac{R_{f}-\frac{W_{0}}{W_{D}^{*}}}{R_{f}-R_{D}}$ and if $R_{f}>1>$ 
$\frac{W_{0}}{W_{D}^{*}}>R_{D}$. That is a high $\lambda_{D}$ induces a loss in the bad state in $\mathrm{t}=2 .{ }^{8}$ The investor makes a neutral result if he chooses to allocate the fraction $\frac{R_{f}-\frac{W_{0}}{W_{D}^{*}}}{R_{f}-R_{D}}$ of his wealth in the risky asset. He makes a gain if he invests a high fraction of his wealth in the risk-free asset and so he preserves his first period gain, i.e. if $\lambda_{D}<\frac{R_{f}-\frac{W_{0}}{W_{D}^{*}}}{R_{f}-R_{D}}$ and $R_{f}>1$ and $\frac{W_{0}}{W_{D}^{*}}, R_{D}<1$.

The relation between the second period wealth and the initial wealth has implications on the form of the objective function in the maximization problem. The maximization problem consists of choosing the optimal fraction of wealth invested in the risky asset, $\lambda_{D}$, subject to the short selling restriction. We write the maximization problem

$$
\max _{1 \geq \lambda_{D} \geq 0} \quad V\left(\lambda_{D}\right)
$$

where

$$
\begin{aligned}
& V=\left\{\begin{array}{ll}
V_{D G L} & \text { if } W_{D U}>W_{0}>W_{D D} \\
V_{D G G} & \text { if } W_{D U} \geq W_{D D}>W_{0} \\
V_{D L L} & \text { if } W_{0}>W_{D U} \geq W_{D D}
\end{array},\right. \\
& V_{D G L}=w(p)\left(W_{D U}-W_{0}\right)^{\alpha}-w(1-p) \beta\left(W_{0}-W_{D D}\right)^{\alpha}, \\
& V_{D G G}=\left(W_{D D}-W_{0}\right)^{\alpha}+w(p)\left(\left(W_{D U}-W_{0}\right)^{\alpha}-\left(W_{D D}-W_{0}\right)^{\alpha}\right) \text {, } \\
& V_{D L L}=-\beta\left(W_{0}-W_{U D}\right)^{\alpha}-w(1-p) \beta\left(\left(W_{0}-W_{D D}\right)^{\alpha}-\left(W_{0}-W_{U D}\right)^{\alpha}\right) \text {, } \\
& W_{D U}=W_{D}^{*}\left(\lambda_{D} R_{U}+\left(1-\lambda_{D}\right) R_{f}\right), W_{D D}=W_{D}^{*}\left(\lambda_{D} R_{D}+\left(1-\lambda_{D}\right) R_{f}\right) \text {, } \\
& \text { and } W_{D}^{*}=W_{0}\left(\lambda_{0}^{*} R_{D}+\left(1-\lambda_{0}^{*}\right) R_{f}\right) \text {. }
\end{aligned}
$$

We use similar arguments as above to determine to optimal asset allocation in $t=1$ after the bad state realized. The detailed calculation can be found in the appendix. The next proposition summarizes our results. Above we have seen, that the ratio between the reference point and the current wealth determines crucially the results. In the case where the stock price went up in the first period this fraction always exceeds unity. In the present case this ratio can be greater, equal or smaller than one. This is the reason for which we structure the proposition in dependence of the first period allocation.

\footnotetext{
${ }^{8}$ Note that the case where $R_{f}>1>R_{D}>\frac{W_{0}}{W_{D}^{*}}$ yields to a contradiction with the requirement that $\lambda_{D}$ has to be between zero and one, i.e. a high $R_{D}$ would not destroy the first period gain.
} 
Proposition 4. Optimal asset allocation in $t=D$ of the investor who measures his performance relative to his initial wealth:

1. For $0 \leq \lambda_{0}^{*} \leq \frac{R_{f}-1}{R_{f}-R_{D}}$ :

(a) If it is optimal to choose a $\lambda_{D}^{*}$ implying a gain in both states, i.e. $W_{D U} \geq W_{D D} \geq W_{0}$, the parameters satisfy $R_{U}>R_{f}>\frac{W_{0}}{W_{D}^{*}} \geq 1$ and if $w(p) \leq \frac{R_{f}-R_{D}}{R_{U}-R_{D}}$, then $\lambda_{D}^{*}=0$. If $w(p)>\frac{R_{f}-R_{D}}{R_{U}-R_{D}}$, then $\lambda_{D}^{*}=\frac{\left(R_{f}-\frac{W_{0}}{W_{D}^{*}}\right)\left(1-\kappa^{\frac{1}{\alpha-1}}\right)}{\left(R_{U}-R_{f}\right) \kappa^{\frac{1}{\alpha-1}}-R_{D}+R_{f}}$, where $\kappa \equiv \frac{w(p)\left(R_{U}-R_{f}\right)}{(1-w(p))\left(R_{f}-R_{D}\right)}$. If further $\frac{R_{f}-\frac{W_{0}}{W_{D}^{*}}}{R_{f}-R_{D}} \geq 1$ and $\kappa^{\frac{1}{\alpha-1}} \leq \frac{R_{D}-\frac{W_{0}}{W_{D}^{*}}}{R_{U}-\frac{W_{0}}{W_{D}^{*}}}$ then $\lambda_{D}^{*}=1$.

(b) If it is optimal to choose $\lambda_{D}$ such that there result a gain and a loss, i.e. $1 \geq \lambda_{D} \geq \frac{R_{f}-\frac{W_{0}}{W_{D}^{*}}}{R_{f}-R_{D}}$, and the parameters satisfy $R_{U}>R_{f} \geq 1>$ $\frac{W_{0}}{W_{D}^{*}}>R_{D}$, and if $V_{G L}\left(\frac{R_{f}-\frac{W_{0}}{W_{D}^{*}}}{R_{f}-R_{D}}\right)>V_{G L}(1)$ then $\lambda_{D G L}^{*}=\frac{R_{f}-\frac{W_{0}}{W_{D}^{*}}}{R_{f}-R_{D}}$, else $\lambda_{D G L}^{*}=1$.

2. For $\lambda_{0}^{*}=\frac{R_{f}-1}{R_{f}-R_{D}}$ :

(a) If it is optimal to choose a $\lambda_{D}^{*}$ implying $W_{D} D \geq W_{0}$ and if $w(p) \leq$ $\frac{R_{f}-R_{D}}{R_{U}-R_{D}}$, then $\lambda_{D}^{*}=0$, else $\lambda_{D}^{*}=\frac{\left(R_{f}-1\right)\left(1-\kappa^{\frac{1}{\alpha-1}}\right)}{\left(R_{U}-R_{f}\right) \kappa^{\frac{1}{\alpha-1}}-R_{D}+R_{f}}$, where $\kappa \equiv$ $\frac{w(p)\left(R_{U}-R_{f}\right)}{(1-w(p))\left(R_{f}-R_{D}\right)}$.

(b) If it is optimal to choose a $\lambda_{D}^{*}$ implying $W_{0} \geq W_{D} D$ and if $V\left(\frac{R_{f}-1}{R_{f}-R_{D}}\right)>V(1)$, then $\lambda_{D}^{*}=\frac{R_{f}-1}{R_{f}-R_{D}}$, else $\lambda_{D}^{*}=1$.

3. $\operatorname{For} \lambda_{0}^{*}=1$ :

(a) If it is optimal to choose a $\lambda_{D}^{*}$ implying a gain in both states, i.e. $W_{D U} \geq W_{D D} \geq W_{0}$, the parameters satisfy $R_{U}>R_{f}>\frac{W_{0}}{W_{D}^{*}}>1$ and if $w(p) \leq \frac{R_{f}-R_{D}}{R_{U}-R_{D}}$, then $\lambda_{D}^{*}=0$. If $w(p)>\frac{R_{f}-R_{D}}{R_{U}-R_{D}}$, then $\lambda_{D}^{*}=\frac{\left(R_{f}-\frac{W_{0}}{W_{D}^{*}}\right)\left(1-\kappa^{\frac{1}{\alpha-1}}\right)}{\left(R_{U}-R_{f}\right) \kappa^{\frac{1}{\alpha-1}}-R_{D}+R_{f}}$, where $\kappa \equiv \frac{w(p)\left(R_{U}-R_{f}\right)}{(1-w(p))\left(R_{f}-R_{D}\right)}$. 
(b) If it is optimal to choose $\lambda_{D}$ such that there result a gain and a loss, i.e. $1 \geq \lambda_{D} \geq \frac{R_{f}-\frac{W_{0}}{W_{D}^{*}}}{R_{f}-R_{D}}$, and the parameters satisfy $R_{U}>$ $R_{f}>\frac{W_{0}}{W_{D}^{*}}>1$. If $V_{G L}\left(\frac{R_{f}-\frac{W_{0}}{W_{D}^{*}}}{R_{f}-R_{D}}\right)>V_{G L}(1)$ then $\lambda_{D G L}^{*}=\frac{R_{f}-\frac{W_{0}}{W_{D}^{*}}}{R_{f}-R_{D}}$, else $\lambda_{D G L}^{*}=1$.

(c) If it is optimal to choose $\lambda_{D}$ such that there result a gain and a loss, i.e. $1 \geq \lambda_{D} \geq \frac{\frac{W_{0}}{W_{D}^{*}}-R_{f}}{R_{U}-R_{f}}$, and the parameters satisfy $R_{U}>\frac{W_{0}}{W_{D}^{*}} \geq$ $R_{f} \geq 1$, then $\lambda_{D}^{*}=\frac{\left(R_{f}-\frac{W_{0}}{W_{D}^{*}}\right)\left(-1-\varphi^{\frac{1}{\alpha-1}}\right)}{\varphi^{\frac{1}{\alpha-1}}\left(R_{U}-R_{f}\right)+R_{D}-R_{f}}$, where $\varphi \equiv \frac{w(p)\left(R_{U}-R_{f}\right)}{w(1-p) \beta\left(R_{f}-R_{D}\right)}$. $\lambda_{D}^{*}=1$ whenever $\varphi^{\frac{1}{\alpha-1}} \leq \frac{\frac{W_{0}}{W_{D}^{*}}-R_{D}}{R_{U}-\frac{W_{0}}{W_{D}^{*}}} \cdot \lambda_{D}^{*}=\frac{\frac{W_{0}}{W_{D}^{*}}-R_{f}}{R_{U}-R_{f}}$ in the special case where $\frac{W_{0}}{W_{D}^{*}}=R_{f}$ which implies that $\lambda_{D}^{*}=0$.

(d) If it is optimal to choose $\lambda_{D}$ such that there result two losses, i.e. $\frac{\frac{W_{0}}{W_{D}^{*}-R_{f}}}{R_{U}-R_{f}} \geq \lambda_{D} \geq 0$ and the parameters satisfy the relation $\frac{W_{0}}{W_{D}^{*}}>R_{f}$, and if $\frac{W_{0}}{W_{D}^{*}}<R_{U}$ and if $V_{L L}(0)>V_{L L}\left(\frac{\frac{W_{0}}{W_{D}^{*}}-R_{f}}{R_{U}-R_{f}}\right)$, then $\lambda_{D L L}^{*}=0$, else $\lambda_{D L L}^{*}=\frac{\frac{W_{0}}{W_{D}^{*}}-R_{f}}{R_{U}-R_{f}}$. If $\frac{W_{0}}{W_{D}^{*}}>R_{U}$ and if $V_{L L}(0)>$ $V_{L L}(1)$, then $\lambda_{D L L}^{*}=0$, else $\lambda_{D L L}^{*}=1$.

If in the first period the agent chooses to invest a low fraction of his wealth in the risky asset he will experience a gain in $t=2$, independently of the state that realizes. However, except for the case where $\lambda_{0}^{*}=0$, the gain in the up state exceeds the gain in the down state. Note that, the condition for $\lambda_{D}^{*}$ to be zero is the same as for $\lambda_{0}^{*}$, namely that the decision weight associated with the up state has to be smaller than the equivalent martingale probability of this state. If this condition is not satisfied then $\lambda_{D}^{*}=\frac{\left(R_{f}-\frac{W_{0}}{W_{D}^{*}}\right)\left(1-\kappa^{\frac{1}{\alpha-1}}\right)}{\left(R_{U}-R_{f}\right) \kappa^{\frac{1}{\alpha-1}}-R_{D}+R_{f}}$. Compared with the solution in $t=0$ we see that the first term in the numerator changes. It is the difference between the gross risk-free return and the ratio between the reference point and the current wealth. This ratio equals one at $t=0 .{ }^{9}$ If the condition for

\footnotetext{
${ }^{9}$ The same observation applies to the case where $\lambda_{D}^{*}=\frac{R_{f}-\frac{W_{0}}{W_{D}^{*}}}{R_{f}-R_{D}}$.
} 
$\lambda_{D}^{*}=0$ is not satisfied and further $\frac{R_{f}-\frac{W_{0}}{W_{D}^{*}}}{R_{f}-R_{D}} \geq 1$ and $\kappa^{\frac{1}{\alpha-1}} \leq \frac{R_{D}-\frac{W_{0}}{W_{D}^{*}}}{R_{U}-\frac{W_{0}}{W_{D}^{*}}}$ then $\lambda_{D}^{*}=1$. The condition $\frac{R_{f}-\frac{W_{0}}{W_{D}^{*}}}{R_{f}-R_{D}} \geq 1$ implies that the boundary for $\lambda_{D}$ implying two gains is bigger than one, where as from $\kappa^{\frac{1}{\alpha-1}} \leq \frac{R_{D}-\frac{W_{0}}{W_{D}^{*}}}{R_{U}-\frac{W_{0}}{W_{D}^{*}}}$ it follows that $\frac{\left(R_{f}-\frac{W_{0}}{W_{D}^{*}}\right)\left(1-\kappa^{\frac{1}{\alpha-1}}\right)}{\left(R_{U}-R_{f}\right) \kappa^{\frac{1}{\alpha-1}}-R_{D}+R_{f}} \geq 1$ so that the optimal allocation in the risky asset is bounded by one, implying $\lambda_{D}^{*}=1$. In the case where it is optimal to choose a high $\lambda_{D}$ the agent choose one of the boundaries, i.e. either $\frac{R_{f}-\frac{W_{0}}{W_{D}^{*}}}{R_{f}-R_{D}}$ or 1 , depending on which of the both choices offers the higher utility.

If the optimal first period choice was $\lambda_{0}^{*}=\frac{R_{f}-1}{R_{f}-R_{D}}$ the investor makes neither a gain nor a loss on his portfolio. The assumptions of a constant investment opportunity set, constant preferences and myopic behavior imply that the investor is confronted with the same optimization problem as in $t=0$ and thus he makes the same choice, i.e. $\lambda_{D}^{*}=\lambda_{0}^{*}=\frac{R_{f}-1}{R_{f}-R_{D}}$.

If in the first period the agent invested his entire wealth in the risky asset and the down state realizes he makes a loss at the end of the first period.

If the parameters satisfy the condition $R_{U}>R_{f}>\frac{W_{0}}{W_{D}^{*}}>1$ the investor can choose to allocate his wealth either such that two gains arise in $t=2$ or such that a gain and a loss may arise. If he chooses to allocate his wealth such that at the end of the second period two gains arise the same statements as above, where two gains in $t=2$ arise, apply. If it is optimal to allocate the first period wealth in a way that there is a possible loss in $t=2$ and the upper boundary applies then $\lambda_{D}^{*}=1$; if the lower boundary applies then $\lambda_{D}^{*}=\frac{R_{f}-\frac{W_{0}}{W_{D}^{*}}}{R_{f}-R_{D}}$. If the parameters satisfy $R_{U}>\frac{W_{0}}{W_{D}^{*}} \geq R_{f} \geq 1$ the investor can choose to allocate his wealth either such that a gain and a loss arise in $t=2$ or such that two losses may arise. If it is optimal to allocate the first period wealth in a way that there is a possible loss in $t=2$ the unconstraint choice is $\lambda_{D}^{*}=\frac{\left(R_{f}-\frac{W_{0}}{W_{D}^{*}}\right)\left(-1-\varphi^{\frac{1}{\alpha-1}}\right)}{\varphi^{\frac{1}{\alpha-1}}\left(R_{U}-R_{f}\right)+R_{D}-R_{f}}$. The upper boundary applies if $\varphi^{\frac{1}{\alpha-1}} \leq \frac{\frac{W_{0}}{W_{D}^{*}}-R_{D}}{R_{U}-\frac{W_{0}}{W_{D}^{*}}}$ so that $\lambda_{D}^{*}=1$. If the lower boundary applies $\lambda_{D}^{*}=\frac{\frac{W_{0}}{W_{D}^{*}}-R_{f}}{R_{U}-R_{f}}$. This arises in the special case where $\frac{W_{0}}{W_{D}^{*}}=R_{f}$ which implies that $\lambda_{D}^{*}=0$. If it is optimal for the investor to choose a $\lambda_{D}$ such that two losses arise in $t=2$ then the 
investor chooses either $\lambda_{D L L}^{*}=0$ or $\lambda_{D L L}^{*}=\frac{\frac{W_{0}}{W_{D}^{*}}-R_{f}}{R_{U}-R_{f}}$. If $\frac{\frac{W_{0}}{W_{D}^{*}-R_{f}}}{R_{U}-R_{f}} \geq 1$ then the optimal fraction invested in the risky asset is bounded by one.

Given the fist period choice, we have to evaluate which of the proposed stationary points is the global maximum or stated differently, we have to answer the question when it is optimal to allocate the wealth in a way that two gains arise in $t=2$, when such that a gain and a loss may arise and when such that two losses arise. In what follows the expression $V_{G G}$ stands for the value function in the case where two gains arise, $V_{G L}$ stands for the value function when no segregation takes place and $V_{L L}$ for the case where two losses arise.

Given it was optimal to choose a $0 \leq \lambda_{0}^{*}<\frac{R_{f}-1}{R_{f}-R_{D}}$ the agent will choose a $\lambda_{D}$ such that there are two gains, i.e. $0 \leq \lambda_{D} \leq \frac{R_{f}-\frac{W_{0}}{W_{D}^{*}}}{R_{f}-R_{D}}$, whenever $V_{G G}(0 \leq$ $\left.\lambda_{D} \leq \frac{R_{f}-\frac{W_{0}}{W_{D}^{*}}}{R_{f}-R_{D}}\right) \geq V_{G L}\left(1 \geq \lambda_{D}>\frac{R_{f}-\frac{W_{0}}{W_{D}^{*}}}{R_{f}-R_{D}}\right)$ or equivalently if

$$
\begin{array}{r}
V_{G G}\left(0<\lambda_{D G G}<\frac{R_{f}-\frac{W_{0}}{W_{D}^{*}}}{R_{f}-R_{D}}\right) \geq V_{G L}\left(\lambda_{D}=\frac{R_{f}-\frac{W_{0}}{W_{D}^{*}}}{R_{f}-R_{D}}\right) \\
\left(\frac{1}{\left(R_{U}-R_{f}\right) \kappa^{\frac{1}{\alpha-1}}+R_{f}-R_{D}}\right)^{\alpha}\left((1-w(p)) \kappa^{\frac{\alpha}{\alpha-1}}+w(p)\right) \geq w(p)\left(\frac{1}{R_{f}-R_{D}}\right)^{\alpha}
\end{array}
$$

and

$$
\begin{array}{r}
V_{G G}\left(0<\lambda_{D G G}<\frac{R_{f}-\frac{W_{0}}{W_{D}^{*}}}{R_{f}-R_{D}}\right) \geq V_{G L}\left(\lambda_{D}=1\right) \\
\left((1-w(p)) \kappa^{\frac{\alpha}{\alpha-1}}+w(p)\right)\left(\frac{\left(R_{f}-\frac{W_{0}}{W_{D}^{*}}\right)\left(R_{U}-R_{D}\right)}{\left(R_{U}-R_{f}\right) \kappa^{\frac{1}{\alpha-1}}+R_{f}-R_{D}}\right)^{\alpha} \\
\geq w(p)\left(R_{U}-\frac{W_{0}}{W_{D}^{*}}\right)^{\alpha}-w(1-p) \beta\left(\frac{W_{0}}{W_{D}^{*}}-R_{D}\right)^{\alpha}
\end{array}
$$

or

$$
\begin{array}{r}
V_{G G}(0) \geq V_{G L}\left(\lambda_{D}=\frac{R_{f}-\frac{W_{0}}{W_{D}^{*}}}{R_{f}-R_{D}}\right) \\
w(p) \leq\left(\frac{R_{f}-R_{D}}{R_{U}-R_{D}}\right)^{\alpha}
\end{array}
$$


and

$$
\begin{array}{r}
V_{G G}(0) \geq V_{G L}\left(\lambda_{D}=1\right) \\
\left(R_{f}-\frac{W_{0}}{W_{D}^{*}}\right)^{\alpha} \geq w(p)\left(R_{U}-\frac{W_{0}}{W_{D}^{*}}\right)^{\alpha}-w(1-p) \beta\left(\frac{W_{0}}{W_{D}^{*}}-R_{D}\right)^{\alpha} .
\end{array}
$$

Given it was optimal for the agent to choose $\lambda_{0}^{*}=\frac{R_{f}-1}{R_{f}-R_{D}}$ in $t=0$, then from the assumptions of a constant investment opportunity set, constant preferences and myopic behavior it follows that in $t=1 \lambda_{D}^{*}=\frac{R_{f}-1}{R_{f}-R_{D}}$ is the global maximum too.

Given it was optimal to choose in $\lambda_{0}^{*}=1$, we have to distinguish two cases: one where the parameters satisfy $R_{U}>R_{f}>\frac{W_{0}}{W_{D}^{*}}>1$ and the other, where they satisfy $R_{U}>\frac{W_{0}}{W_{D}^{*}} \geq R_{f} \geq 1$. If $R_{U}>R_{f}>\frac{W_{0}}{W_{D}^{*}}>1$ then the same conditions as in the case where the agent chose $0 \leq \lambda_{0}^{*}<\frac{R_{f}-1}{R_{f}-R_{D}}$ apply.

If $R_{U}>\frac{W_{0}}{W_{D}^{*}} \geq R_{f} \geq 1$ the investor chooses to allocate his wealth such that he makes a gain in $t=D U$ and a loss in $t=D D$ whenever $V_{G L}(1 \geq$ $\left.\lambda_{D} \geq \frac{\frac{W_{0}}{W_{D}^{0}}-R_{f}}{R_{U}-R_{f}}\right) \geq V_{L L}\left(\frac{\frac{W_{0}}{W_{D}}-R_{f}}{R_{U}-R_{f}} \geq \lambda_{D} \geq 0\right)$ or equivalently if

$$
\begin{array}{r}
V_{G L}\left(1>\lambda_{D}>\frac{\frac{W_{0}}{W_{D}^{*}}-R_{f}}{R_{U}-R_{f}}\right) \geq V_{L L}(0) \\
w(p)\left(\frac{\left(R_{U}-R_{D}\right)\left(W_{0}-W_{D}^{*} R_{f}\right)}{\varphi^{\frac{1}{\alpha-1}}\left(R_{U}-R_{f}\right)+R_{D}-R_{f}}\right)^{\alpha} \\
-w(1-p) \beta\left(\frac{\left(R_{U}-R_{D}\right) \varphi^{\frac{1}{\alpha-1}}\left(W_{0}-W_{D}^{*} R_{f}\right)}{\varphi^{\frac{1}{\alpha-1}}\left(R_{U}-R_{f}\right)+R_{D}-R_{f}}\right)^{\alpha} \\
\geq-\beta\left(W_{0}-W_{D}^{*} R_{f}\right)^{\alpha}
\end{array}
$$


and

$$
\begin{array}{r}
V_{G L}\left(1>\lambda_{D}>\frac{\frac{W_{0}}{W_{D}^{*}}-R_{f}}{R_{U}-R_{f}}\right) \geq V_{L L}\left(\frac{\frac{W_{0}}{W_{D}^{*}}-R_{f}}{R_{U}-R_{f}}\right) \\
w(p)\left(\frac{\left(R_{U}-R_{D}\right)\left(W_{0}-W_{D}^{*} R_{f}\right)}{\varphi^{\frac{1}{\alpha-1}}\left(R_{U}-R_{f}\right)+R_{D}-R_{f}}\right)^{\alpha} \\
-w(1-p) \beta\left(\frac{\left(R_{U}-R_{D}\right) \varphi^{\frac{1}{\alpha-1}}\left(W_{0}-W_{D}^{*} R_{f}\right)}{\varphi^{\frac{1}{\alpha-1}}\left(R_{U}-R_{f}\right)+R_{D}-R_{f}}\right)^{\alpha} \\
\geq-w(1-p) \beta\left(\frac{\left(R_{U}-R_{D}\right)\left(W_{0}-W_{D}^{*} R_{f}\right)}{R_{U}-R_{f}}\right)^{\alpha}
\end{array}
$$

or

$$
\begin{array}{r}
V_{G L}(1) \geq V_{L L}(0) \\
w(p)\left(W_{D}^{*} R_{U}-W_{0}\right)^{\alpha}-w(1-p) \beta\left(W_{0}-W_{D}^{*} R_{D}\right)^{\alpha} \geq-\beta\left(W_{0}-W_{D}^{*} R_{f}\right)^{\alpha}
\end{array}
$$

and

$$
\begin{array}{r}
V_{G L}(1) \geq V_{L L}\left(\frac{\frac{W_{0}}{W_{D}^{*}}-R_{f}}{R_{U}-R_{f}}\right) \\
w(p)\left(W_{D}^{*} R_{U}-W_{0}\right)^{\alpha}-w(1-p) \beta\left(W_{0}-W_{D}^{*} R_{D}\right)^{\alpha} \\
\geq-w(1-p) \beta\left(\frac{\left(R_{U}-R_{D}\right)\left(W_{0}-W_{D}^{*} R_{f}\right)}{R_{U}-R_{f}}\right)^{\alpha}
\end{array}
$$

or

$$
\begin{array}{r}
V_{G L}(0) \geq V_{L L}(0) \\
0 \geq-\beta\left(W_{0}-W_{D}^{*} R_{f}\right)^{\alpha}
\end{array}
$$

and

$$
\begin{array}{r}
V_{G L}(0) \geq V_{L L}\left(\frac{\frac{W_{0}}{W_{D}^{*}}-R_{f}}{R_{U}-R_{f}}\right) \\
0 \geq-w(1-p) \beta\left(\frac{\left(R_{U}-R_{D}\right)\left(W_{0}-W_{D}^{*} R_{f}\right)}{R_{U}-R_{f}}\right)^{\alpha} .
\end{array}
$$

Note that equation (20) is always satisfied in the relevant case, namely for all $\beta$ and $W_{D}^{*}$ positive and for $\frac{W_{0}}{W_{D}^{*}} \geq R_{f}$. Equation (21) is satisfied for 
positive $w(1-p), \beta$ and $W_{D}^{*}$ and for $\frac{W_{0}}{W_{D}^{*}} \geq R_{f}$ and in absence of arbitrage, i.e $R_{U}>R_{f}>R_{D}$. From this it follow that after $\lambda_{0}^{*}=1$ in the case where $R_{U}>\frac{W_{0}}{W_{D}^{*}} \geq R_{f} \geq 1>R_{D}$ to allocate the wealth in a way that a gain and a loss arises and in particular $\lambda_{D}^{*}=0$ dominates the case where the agent chooses to allocate his wealth in a way that two losses arise in $t=2$. Note further that in the case where the agent chooses to allocate such that a gain and a loss arise, $\lambda_{D}^{*}=0$ is a special case and arises only if $R_{f}=\frac{W_{0}}{W_{D}^{*}}$, else it is optimal to choose a $\lambda_{D}^{*}>0$, which offers a higher value, so that it is never optimal to allocate the wealth in a way that two losses arise.

These conditions involve the parameters of the agent's preferences, $\alpha, \beta$ and $w(p)$, as well as the parameters of the financial market, i.e. the possible returns and the probabilities for the possible states, and the first period optimal choices. Since too many parameters are involved it is not possible to find closed form solutions for these inequalities of higher order. Therefore we present numerical results for the optimal allocation of wealth in $t=D$.

Figure 5 shows the optimal $\lambda_{0}^{*}$ and optimal $\lambda_{D}^{*}$ for different stock returns $R_{U}$ and $R_{D}$. $R_{U}$ ranges from 1 to 2 and $R_{D}$ from 0 to 1 . The risk-free rate is fixed at $0.1, \alpha=0.88, \beta=2.25$ and $\gamma=0.65$. The probability of the good state equals 0.5 . The results for the second period are consistent with optimal first period behavior.

In the corner, where the return of the stock in the good state is very close to the risk-free return and where the gross return in the down state is close to zero the optimal asset allocation in $t=1$ after the stock price has fallen in the first period is $\lambda_{D}^{*}=0$. The reason is that in this case the risk-free investment is more attractive. When the risky assets offers more attractive returns the agent invest gradually more in the stock. When the risky asset is very attractive, i.e. nearly doubles his value in the good state and practically does not loose its value in the bad state, the agent invests his entire wealth in it. Note that the domain, where it is optimal to invest the whole wealth in the risk-free asset is the same in all periods and in all states, i.e. in $t=0$, $t=U$ and $t=D$. Further we observe, that $\lambda_{D}^{*}$ goes up steeper than $\lambda_{0}^{*}$ in the domain where the agent experiences a gain in his wealth in the first period, i.e in the domain where $0 \leq \lambda_{0}^{*}<\frac{R_{f}-1}{R_{f}-R_{D}}$. If in the first period it was optimal to invest the fraction for $\lambda_{0}^{*}=\frac{R_{f}-1}{R_{f}-R_{D}}$ in the risky asset it follows, that in $t=1$ the agent makes a zero gain after the stock declines. Since all the parameters are constant over time and the agents acts myopically it follows that in this case her decision in $t=D$ will be the same as in $t=0$. 

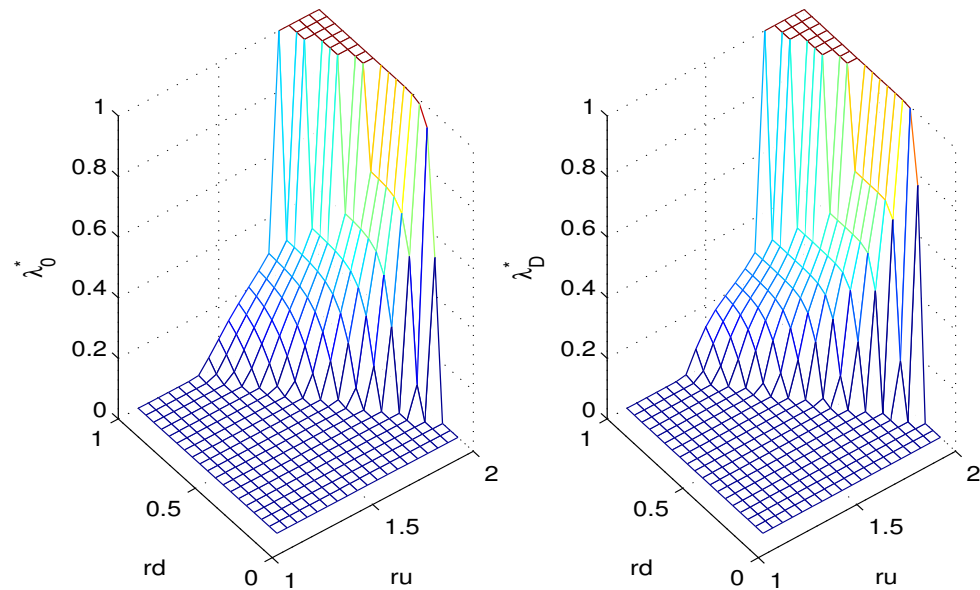

Figure 5: $\lambda_{0}^{*}$ and $\lambda_{D}^{*}$ for different stock returns $R_{U}$ and $R_{D}$. The risk-free rate is fixed at $0.1, p=0.5, \alpha=0.88, \beta=2.25$ and $\gamma=0.65$.

If the investor chose in $t=0$ to allocate his total wealth in the risky asset in the first period, then he does so in the second period too.

Recapitulating we can say that in $t=0$ the agent invests all her wealth in the risk-free bond when it is relatively more attractive than the risky stock. This is the case when the decision weight associated with the good state is lower than the equivalent martingale probability of this state. The more the risky asset gains in attractiveness the more the agent is willing to invest in it. This change occurs gradually. Remember that choosing a low $\lambda_{0}$ produces two gains in $t=1$. Once the strategy that implies a zero gain in the bad state in $t=1$ is reached, the optimal strategy for even more attractive securities is to invest the whole wealth in the risky asset.

In $t=1$ the portfolio decision looks quite the same as in $t=0$ : if the decision weight for the up state is smaller than the associated equivalent martingale probability, the investor chooses to allocate his entire wealth in the risk-free bond. Note that this condition is the same in both periods and in both states. The more attractive the stock is, the higher the fraction invested in it. In particular we do not observe any "reversal" in strategies in $t=1$. However we observe that after the stock went up in the first period the agent never invests lower fraction in the risky asset than he did in $t=0$. 
If the price of the stock declined in the first period the agent invests the same or a higher fraction of his wealth in the risky asset whenever he experienced a gain in his portfolio, i.e. if he chose to invest a low fraction of his wealth in $t=0$ in the risky asset. After a loss in wealth, note that this case occurs after the agent chose in $t=0$ to invest his entire wealth in the risky asset, he allocates again the whole wealth in the risky investment opportunity.

\section{Conclusions}

In the finance literature there are conflicting results about the effect of prior outcomes on risk-taking behavior in subsequent periods. Some authors report that investors are risk-seeking after a gain and risk averse after a loss while other studies find the opposite behavior.

In this article we consider a two period model for portfolio choice in a stylized financial market where the investor's preferences are described by prospect theory. Following Tversky and Kahneman (1992) we assume a two part power function, reflecting loss aversion and asymmetric risk-taking behavior. Note, that we take the segregation of riskless alternatives during the editing phase explicitly into account.

Our point of interest is the second period behavior of the investor conditional on the stock price movement in the first period. In particular, we ask if we can explain the behavior of an investor prone to the disposition effect or prone to the house money effect. In our model we define the risk-taking behavior in terms of portfolio weights: an investor who chooses a riskier strategy after a gain and a safer strategy after a loss is a house money investor. If the agent invests less in the risky asset after the stock appreciated and more after it depreciated, we call him a disposition investor.

Whereas in the first period all agents solve the same maximization problem, the second period decision depends on the reference point relative to which the agent measures his second period gains and losses. In our framework there are two prominent candidates for reference points: the initial wealth or the current wealth. We analyze both cases.

For the investor who measures his performance relative to his initial wealth we find the following results: in the first period the agent invests all her wealth in the risk-free bond when it is relatively more attractive than

the risky stock. This is the case when the decision weight associated with the 
good state is lower than the equivalent martingale probability of this state. The more the risky asset gains in attractiveness the more the agent is willing to invest in it. This change occurs gradually. The optimal strategy for a very attractive securities is to invest the whole wealth in the risky asset.

In the second period the portfolio decision looks similar to the first period: if the decision weight for the up state is smaller than the associated equivalent martingale probability, the investor chooses to allocate his entire wealth in the risk-free bond. Note that this condition is the same in both periods and in both states. The more attractive the stock, the higher the fraction invested in it. We observe that after the stock went up in the first period the agent never invests a lower fraction in the risky asset than he did in the first period. If the price of the stock declined in the first period the agent invests the same or a higher fraction of his wealth in the risky asset whenever he experienced a gain in his portfolio. After a loss in wealth, which occurs after the agent chose in the first period to invest his entire wealth in the risky asset, he allocates again the whole wealth in the risky investment opportunity. This case arises only when the stock is very attractive.

Our findings are that the changes in portfolio weights depend crucially on the ratio between the reference point and the current wealth, and therefore only indirectly on the performance of the stock.

So even when considering explicitly the asymmetric risk-taking behavior of the investor, a standard explication for the disposition behavior, our investor is not prone to the disposition effect. In particular he chooses a riskier strategy after the stock appreciated. His behavior is essentially determined by loss aversion: a first period gain in wealth cushions possible future losses, so that the investor chooses a riskier strategy.

Further we observe that it is never optimal for the investor to allocate his wealth in a way, that in the next period in both states a loss will occur. That is the investor never puts himself in the loss domain where he is risk-seeking. His loss aversion prevents him to get that deep into the loss region that in the next period he will be confronted with losses only. If the agent incurs a loss in his portfolio it is because in the first period he invested all his wealth in the risky asset. He does so only when the stock is very attractive, i.e. has a very small loss potential and a very high upside potential. After the bad state realized and the investor incurred a loss in his portfolio, the stock is still very attractive and offers him the possibility to compensate the loss from the previous period. This is the reason why the agent keeps on holding this stock. 
Our results are consistent with the house money effect in the sense that investors follow, in relative terms, a riskier strategy after a first period gain. Note that a higher fraction invested in the risky asset, after the stock appreciated, can be consistent with both holding more and less units of the risky asset. So that the results of our model can be consistent, in absolute terms, with a disposition investor's behavior. Our model explains why investor keep on holding, or even buy, loosing investments: either because the stock is very attractive and offers a high chance to undo the loss experienced in the portfolio -this is consistent with the break-even effect- or either because the gain in the portfolio makes possible future losses more bearable. Empirical tests, taking the relation between stock prices and the portfolio performance into account, have to be conducted to asses the validity of our predictions. An interesting issue is whether the disposition effect arises only in terms of units bought and sold or also in terms of portfolio weights.

Possible extensions are to include narrow framing into our model or to use a dynamic optimization approach. However we do not think that dynamic optimization is an appropriate way to describe the behavior of individual investors. An other possibility would be to use another functional form for the utility function, which still has the properties Kahneman and Tversky found in their experimental evidence. E.g. a piece wise exponential utility function as proposed by De Giorgi, Hens and Levy (2004) or Kyle et al. (2004).

The agent who measures his gains and losses relative to his actual wealth solves in each period the same maximizing problem. Therefore he allocates in each period the same fraction of his wealth to the risky asset, that is he plays a fix-mix strategy. An open question remains, if a fix-mix strategy that implies a rebalancing after each stock price movement can be seen as a cause for the disposition effect.

\section{A Appendix}

\section{A.1 Optimal Asset Allocation in $\mathrm{t}=0$}




\section{Proof of Proposition 1}

Since the editing of the prospects implies different forms of the value function we have to treat the two possible cases separately. First consider the case where the investor chooses to allocate his wealth such that only gains are possible. This case arises when the investor chooses a $\lambda_{0}^{*} \in\left[0, \frac{R_{f}-1}{R_{f}-R_{D}}\right]$. Note that in the case where $\lambda_{0}^{*}=\frac{R_{f}-1}{R_{f}-R_{D}}$ neither a gain nor a loss occurs. However, in this section we treat this case for methodological reasons as a zero gain.

The objective function writes

$$
\begin{aligned}
V_{0 G G}\left(\lambda_{0}\right)= & v\left(W_{D}-W_{0}\right)+w(p)\left(v\left(W_{U}-W_{0}\right)-v\left(W_{D}-W_{0}\right)\right) \\
= & (1-w(p))\left(W_{0}\left(\left(R_{D}-R_{f}\right) \lambda_{0}+R_{f}-1\right)\right)^{\alpha} \\
& +w(p)\left(W_{0}\left(\left(R_{U}-R_{f}\right) \lambda_{0}+R_{f}-1\right)\right)^{\alpha} .
\end{aligned}
$$

First consider the case where the constraints do not bind. The first and second derivative of the the objective function are

$$
\begin{array}{r}
V_{0 G G}^{\prime}=+w(p) \alpha\left(W_{0}\left(\left(R_{U}-R_{f}\right) \lambda_{0}+R_{f}-1\right)\right)^{\alpha-1} W_{0}\left(R_{U}-R_{f}\right) \\
+(1-w(p)) \alpha\left(W_{0}\left(\lambda_{0}\left(R_{D}-R_{f}\right)+R_{f}-1\right)\right)^{\alpha-1} W_{0}\left(R_{D}-R_{f}\right) \\
V_{0 G G}^{\prime \prime}=\alpha(\alpha-1) w(p)\left(W_{0}\left[\left(R_{U}-R_{f}\right) \lambda_{0}+R_{f}-1\right]\right)^{\alpha-2} W_{0}^{2}\left(R_{u}-R_{f}\right)^{2} \\
+\alpha(\alpha-1)(1-w(p))\left(W_{0}\left(\lambda_{0}\left(R_{D}-R_{f}\right)+R_{f}-1\right)\right)^{\alpha-2} W_{0}^{2}\left(R_{D}-R_{f}\right)^{2} \\
=\alpha(\alpha-1) W_{0}^{2}\left((1-w(p))\left(W_{0}\left(\lambda_{0}\left(R_{D}-R_{f}\right)+R_{f}-1\right)\right)^{\alpha-2}\left(R_{f}-R_{d}\right)^{2}\right. \\
\left.+w(p)\left(W_{0}\left(\left(R_{U}-R_{f}\right) \lambda_{0}+R_{f}-1\right)\right)^{\alpha-2}\left(R_{U}-R_{f}\right)^{2}\right) .
\end{array}
$$

For $0 \leq w(p) \leq 1, R_{U}>R_{f} \geq 1>R_{D}, 0 \leq \alpha \leq 1$ and $W_{0}>0$ the second derivative of the objective function is smaller than zero, implying that the value function for the case where only gains arise is concave.

Solving the first order condition yields the optimal portfolio allocation for 
the unconstraint case

$$
\begin{aligned}
& \lambda_{0}^{*}=\frac{\left(R_{f}-1\right)\left(1-\kappa^{\frac{1}{\alpha-1}}\right)}{\left(R_{U}-R_{f}\right) \kappa^{\frac{1}{\alpha-1}}+R_{f}-R_{D}}, \\
& \text { where } \kappa \equiv \frac{w(p)\left(R_{U}-R_{f}\right)}{(1-w(p))\left(R_{f}-R_{D}\right)} .
\end{aligned}
$$

From the concavity of the objective function it follows that $\lambda_{0}^{*}$ is a maximum.

Next consider the case where the lower boundary, i.e. $\lambda_{0}=0$, is binding. Using the concavity property it follows that this cases arises whenever the unconstraint $\lambda_{0}^{*}$ is negative or

$$
\begin{array}{r}
\left(R_{f}-1\right)\left(1-\kappa^{\frac{1}{\alpha-1}}\right) \leq 0 \\
w(p) \leq \frac{R_{f}-R_{D}}{R_{U}-R_{D}} .
\end{array}
$$

When the parameters of the problem satisfy this condition the optimal fraction invested in the risky asset is zero. Note that $\frac{R_{f}-R_{D}}{R_{U}-R_{D}}$ is greater than zero and smaller than one in absence of arbitrage. We can interpret this term as the ratio between the difference in returns between the riskless asset and the stock's return in the bad state and the spread between the stock's possible returns. The higher the stock return in the good state ceteris paribus, the lower the chance that $\lambda_{0}^{*}$ equals zero. The higher the risk-free rate, the higher the chance that $\lambda_{0}^{*}$ equals zero.

The upper boundary applies in absence of arbitrage in the special case where $R_{f}=1$. To see this, note that

$$
\begin{aligned}
\lambda_{0}^{*} & \leq \frac{R_{f}-1}{R_{f}-R_{D}} \\
\frac{\left(R_{f}-1\right)\left(1-\kappa^{\frac{1}{\alpha-1}}\right)}{\left(R_{U}-R_{f}\right) \kappa^{\frac{1}{\alpha-1}}-\left(R_{D}-R_{f}\right)} & \leq \frac{R_{f}-1}{R_{f}-R_{D}} .
\end{aligned}
$$

So that for $R_{f}>1$

$$
\begin{aligned}
\frac{1-\kappa^{\frac{1}{\alpha-1}}}{\left(R_{U}-R_{f}\right) \kappa^{\frac{1}{\alpha-1}}-\left(R_{D}-R_{f}\right)}<\frac{1}{R_{f}-R_{D}} \\
R_{D} \kappa^{\frac{1}{\alpha-1}}<R_{U} \kappa^{\frac{1}{\alpha-1}} \\
R_{D}<R_{U}
\end{aligned}
$$


which is satisfied in absence of arbitrage. For $R_{f}=1 \lambda_{0}^{*}=0$ so that $\lambda_{0}^{*}=\frac{R_{f}-1}{R_{f}-R_{D}}$, for all parameters.

Note that in the domain where only gains are possible, the value function is concave. Since the opportunity set is concave, following the Kuhn-Tucker theorem, the stationary point is a global maximizer.

The other case, i.e. the one without segregation, arises when the agent chooses to allocate his wealth in a way such that there is a possible loss at the end of the next period. This cases arises when the investor chooses a $\lambda_{0}^{*} \in\left[\frac{R_{f}-1}{R_{f}-R_{D}}, 1\right]$. Note that strictly speaking $\lambda_{0}^{*}=\frac{R_{f}-1}{R_{f}-R_{D}}$ implies neither a loss, nor a gain. However, for methodological reasons, we treat it in this section as a zero loss. This does not alter our results. The objective function writes

$$
\begin{aligned}
V_{0 G L}\left(\lambda_{0}\right)= & w(p) v\left(W_{U}\left(\lambda_{0}\right)-W_{0}\right)+w(1-p) v\left(W_{D}\left(\lambda_{0}\right)-W_{0}\right) \\
= & w(p)\left(W_{0}\left(\left(R_{U}-R_{f}\right) \lambda_{0}+R_{f}-1\right)\right)^{\alpha} \\
& +w(1-p)(-\beta)\left(W_{0}\left(1-\left(R_{D}-R_{f}\right) \lambda_{0}-R_{f}\right)\right)^{\alpha} .
\end{aligned}
$$

First consider the case where the constraints do not bind. The first order condition writes

$$
\begin{gathered}
\alpha w(1-p) \beta\left(W_{0}\left(1-\lambda_{0}\left(R_{D}-R_{f}\right)-R_{f}\right)\right)^{\alpha-1} W_{0}\left(R_{D}-R_{f}\right) \\
+\alpha w(p)\left(W_{0}\left(\lambda_{0}\left(R_{U}-R_{f}\right)+R_{f}-1\right)\right)^{\alpha-1} W_{0}\left(R_{U}-R_{f}\right)=0 .
\end{gathered}
$$

Solving the first order condition yields the stationary point for the optimal portfolio allocation for the unconstraint case

$$
\begin{aligned}
& \lambda_{0}^{*}=\frac{\left(R_{f}-1\right)\left(-1-\varphi^{\frac{1}{\alpha-1}}\right)}{\varphi^{\frac{1}{\alpha-1}}\left(R_{U}-R_{f}\right)+R_{D}-R_{f}}, \\
& \text { where } \varphi \equiv \frac{w(p)\left(R_{U}-R_{f}\right)}{w(1-p) \beta\left(R_{f}-R_{D}\right)} .
\end{aligned}
$$

Note that in absence of arbitrage $\varphi>0$ for all $0<p<1$ and that $\lambda_{0}^{*}$ is positive whenever $\varphi^{\frac{1}{1-\alpha}}>\frac{R_{U}-R_{f}}{R_{f}-R_{D}}$. This follows from

$$
\begin{array}{r}
\lambda_{0}^{*}=\frac{\left(R_{f}-1\right)\left(-1-\varphi^{\frac{1}{\alpha-1}}\right)}{\varphi^{\frac{1}{\alpha-1}}\left(R_{U}-R_{f}\right)+R_{D}-R_{f}} \\
\lambda_{0}^{*}>0 .
\end{array}
$$


Note that the numerator is negative, since the second multiplicand is.

$$
\begin{array}{r}
\varphi^{\frac{1}{\alpha-1}}\left(R_{U}-R_{f}\right)+R_{D}-R_{f}<0 \\
\varphi^{\frac{1}{\alpha-1}}<\frac{R_{f}-R_{D}}{R_{U}-R_{f}} \\
\varphi^{\frac{1}{1-\alpha}}>\frac{R_{U}-R_{f}}{R_{f}-R_{D}} .
\end{array}
$$

The stationary point $\lambda_{0}^{*}=\frac{\left(R_{f}-1\right)\left(-1-\varphi^{\frac{1}{\alpha-1}}\right)}{\varphi^{\frac{1}{\alpha-1}}\left(R_{U}-R_{f}\right)+R_{D}-R_{f}}$ is an unconstraint maximum if the second derivative of the objective function at this point is negative.

$$
\begin{aligned}
V_{0 G L}^{\prime \prime}\left(\lambda_{0}\right)= & \alpha(\alpha-1) w(p)\left(W_{0}\left(\lambda_{0}\left(R_{U}-R_{f}\right)+R_{f}-1\right)\right)^{\alpha-2} W_{0}^{2}\left(R_{U}-R_{f}\right)^{2} \\
& -\alpha(\alpha-1) w(1-p)(\beta)\left(W_{0}\left(1-\lambda_{0}\left(R_{D}-R_{f}\right)-R_{f}\right)\right)^{\alpha-2} \\
& W_{0}^{2}\left(R_{D}-R_{f}\right)^{2} ;
\end{aligned}
$$
if

note that $\alpha-1$ is smaller than zero. The stationary point $\lambda_{0}^{*}$ is a maximum

$$
\begin{array}{r}
\left.0>-w(p)\left(W_{0}\left(\lambda_{0}^{*}\left(R_{U}-R_{f}\right)+R_{f}-1\right)\right)^{\alpha-2}\left(R_{U}^{\prime \prime}-R_{f}\right)^{2}\right)<0 \\
+w(1-p)(\beta)\left(W_{0}\left(1-\lambda_{0}^{*}\left(R_{D}-R_{f}\right)-R_{f}\right)\right)^{\alpha-2}\left(R_{f}-R_{D}\right)^{2} \\
\frac{\left(1-\frac{\left(R_{D}-R_{f}\right)\left(R_{f}-1\right)\left(-1-\varphi^{\frac{1}{\alpha-1}}\right)}{\varphi^{\frac{1}{\alpha-1}}\left(R_{U}-R_{f}\right)+R_{D}-R_{f}}-R_{f}\right)^{\alpha-2}}{\left(\frac{\left(R_{U}-R_{f}\right)\left(R_{f}-1\right)\left(-1-\varphi^{\frac{1}{\alpha-1}}\right)}{\varphi^{\frac{1}{\alpha-1}}\left(R_{U}-R_{f}\right)+R_{D}-R_{f}}+R_{f}-1\right)^{\alpha-2}}<\varphi \frac{R_{U}-R_{f}}{R_{f}-R_{D}} \\
\varphi^{\frac{1}{1-\alpha}}<\frac{R_{U}-R_{f}}{R_{f}-R_{D}} .
\end{array}
$$

However, as we shown above, for $\lambda_{0}^{*}$ to be positive we require $\varphi^{\frac{1}{1-\alpha}}>$ $\frac{R_{U}-R_{f}}{R_{f}-R_{D}}$, implying, that $\lambda_{0}^{*}$ in fact is a minimum, given the requirement of non-negativity. Since the stationary point is unique this result implies that 
in the domain of positive $\lambda_{0}$ the value function for the case where a gain and a loss arise is convex.

The theorem of Weierstrass states that if the opportunity set is compact (i.e. closed and bounded) and nonenmpty and the objective function is continuous on the opportunity set, the objective has a global maximum either in the interior or on the boundary of the opportunity set. Thus, in our case the maximum is at one of the boundaries. So we can infer that in the case where the investor chooses to allocate his wealth in a way that there is a possible loss in the next period the optimal allocation is

$$
\lambda_{0}^{*}=\left\{\begin{array}{ll}
\frac{R_{f}-1}{R_{f}-R_{D}} & \text { if } V_{0 G L}\left(\frac{R_{f}-1}{R_{f}-R_{D}}\right)>V_{0 G L}(1) \\
1 & \text { if } V_{0 G L}(1)>V_{0 G L}\left(\frac{R_{f}-1}{R_{f}-R_{D}}\right)
\end{array} .\right.
$$




\section{A.2 Optimal Asset Allocation of the Investor who's Reference Point is his Current Wealth}

Proof of Proposition 2

This result follows from the fact that the preference and market parameters are constant over time, implying that the investment opportunity set is constant, and from the fact that the investor acts myopically.

\section{A.3 Optimal Asset Allocation in $\mathrm{t}=\mathrm{U}$ of the Investor who's Reference Point is his Initial Wealth}

\section{Proof of Proposition 3}

This proof follows the proof of proposition 1. Since the editing of the prospects implies different forms of the value function we have to treat the two possible cases separately. First consider the case where the investor chooses to allocate his wealth such that only gains are possible. This case arises when the investor chooses a $\lambda_{U}^{*} \in\left[0, \frac{R_{f}-\frac{W_{0}}{W_{U}^{*}}}{R_{f}-R_{D}}\right]$. Note that in the case where $\lambda_{0}^{*}=\frac{R_{f}-\frac{W_{0}}{W_{U}^{*}}}{R_{f}-R_{D}}$ neither a gain nor a loss occurs. However, in this section we treat this case for methodological reasons as a zero gain.

The objective function writes

$$
\begin{aligned}
V_{U G G}\left(\lambda_{U}\right)= & v\left(W_{U D}-W_{0}\right)+w(p)\left(v\left(W_{U U}-W_{0}\right)-v\left(W_{U D}-W_{0}\right)\right) \\
= & (1-w(p))\left(W_{U}^{*}\left(\left(R_{D}-R_{f}\right) \lambda_{U}+R_{f}\right)-W_{0}\right)^{\alpha} \\
& +w(p)\left(W_{U}^{*}\left(\left(R_{U}-R_{f}\right) \lambda_{U}+R_{f}\right)-W_{0}\right)^{\alpha}
\end{aligned}
$$

First consider the case where the constraints do not bind. The first and 
second derivative of the the objective function are

$$
\begin{gathered}
V_{U G G}^{\prime}=+\alpha w(p)\left(W_{U}^{*}\left(\left(R_{U}-R_{f}\right) \lambda_{U}+R_{f}\right)-W_{0}\right)^{\alpha-1} W_{U}^{*}\left(R_{U}-R_{f}\right) \\
+\alpha(1-w(p))\left(W_{U}^{*}\left(\lambda_{U}\left(R_{D}-R_{f}\right)+R_{f}\right)-W_{0}\right)^{\alpha-1} W_{U}^{*}\left(R_{D}-R_{f}\right) \\
V_{U G G}^{\prime \prime}=\left((1-w(p))\left(W_{U}^{*}\left(\lambda_{U}\left(R_{D}-R_{f}\right)+R_{f}\right)-W_{0}\right)^{\alpha-2}\left(R_{f}-R_{D}\right)^{2}\right. \\
\left.+w(p)\left(W_{U}^{*}\left(\left(R_{U}-R_{f}\right) \lambda_{U}+R_{f}\right)-W_{0}\right)^{\alpha-2}\left(R_{U}-R_{f}\right)^{2}\right) \alpha(\alpha-1) W_{U}^{* 2} .
\end{gathered}
$$

For $0 \leq w(p) \leq 1, R_{U}>R_{f} \geq 1>R_{D}, 0<\alpha<1$ and $W_{0}>0$ the second derivative of the objective function is negative, implying that the value function for the case where only gains arise is concave.

Solving the first order condition yields the optimal portfolio allocation for the unconstraint case

$$
\begin{gathered}
\lambda_{U}^{*}=\frac{\left(R_{f}-\frac{W_{0}}{W_{U}^{*}}\right)\left(1-\kappa^{\frac{1}{\alpha-1}}\right)}{\left(R_{U}-R_{f}\right) \kappa^{\frac{1}{\alpha-1}}+R_{f}-R_{D}}, \\
\text { where } \kappa \equiv \frac{w(p)\left(R_{U}-R_{f}\right)}{(1-w(p))\left(R_{f}-R_{D}\right)} .
\end{gathered}
$$

From the concavity of the objective function it follows that $\lambda_{U}^{*}$ is a maximum.

Next consider the case where the lower boundary, i.e. $\lambda_{U}=0$, is binding. Using the concavity property it follows that this cases arises whenever the unconstraint $\lambda_{U}^{*}$ is negative or $w(p) \leq \frac{R_{f}-R_{D}}{R_{U}-R_{D}}$.

If the upper boundary is binding then $\lambda_{U}^{*}=\frac{R_{f}-\frac{W_{0}}{W_{U}^{*}}}{R_{f}-R_{D}}$. In the case where $\frac{R_{f}-\frac{W_{0}}{W_{U}^{*}}}{R_{f}-R_{D}} \geq 1 \lambda_{U}^{*}=1$ when ever $\kappa^{\frac{1}{\alpha-1}} \leq \frac{R_{D}-\frac{W_{0}}{W_{U}^{*}}}{R_{U}-\frac{W_{0}}{W_{U}^{*}}}$.

The other case, i.e. the one without segregation, arises when the agent chooses to allocate his wealth in a way such that there is a possible loss at the end of the next period. This case arises when the investor chooses a $\lambda_{0}^{*} \in\left[\frac{R_{f}-\frac{W_{0}}{W_{U}^{*}}}{R_{f}-R_{D}}, 1\right] .{ }^{10}$ Note that strictly speaking $\lambda_{U}^{*}=\frac{R_{f}-\frac{W_{0}}{W_{U}^{*}}}{R_{f}-R_{D}}$ implies neither

${ }^{10}$ This is only consistent with the short-selling restriction when $\frac{R_{f}-\frac{W_{0}}{W_{U}^{W}}}{R_{f}-R_{D}} \leq 1$. 
a loss, nor a gain. However, for methodological reasons, we treat it in this section as a zero loss. This does not alter our results. The objective function writes

$$
\begin{aligned}
V_{U G L}\left(\lambda_{U}\right)= & w(p)\left(W_{U}^{*}\left(\left(R_{U}-R_{f}\right) \lambda_{U}+R_{f}\right)-W_{0}\right)^{\alpha} \\
& +w(1-p)(-\beta)\left(W_{0}-W_{U}^{*}\left(\left(R_{D}-R_{f}\right) \lambda_{U}+R_{f}\right)\right)^{\alpha} .
\end{aligned}
$$

First consider the case where the constraints do not bind. The first order condition writes

$$
\begin{aligned}
& \alpha w(1-p) \beta\left(W_{0}-W_{U}^{*}\left(\lambda_{U}\left(R_{D}-R_{f}\right)+R_{f}\right)\right)^{\alpha-1} W_{U}^{*}\left(R_{D}-R_{f}\right) \\
& +\alpha w(p)\left(W_{U}^{*}\left(\lambda_{U}\left(R_{U}-R_{f}\right)+R_{f}\right)-W_{0}\right)^{\alpha-1} W_{U}^{*}\left(R_{U}-R_{f}\right)=0 .
\end{aligned}
$$

Solving the first order condition yields the stationary point for the optimal portfolio allocation for the unconstraint case

$$
\begin{gathered}
\lambda_{U}^{*}=\frac{\left(R_{f}-\frac{W_{0}}{W_{U}^{*}}\right)\left(-1-\varphi^{\frac{1}{\alpha-1}}\right)}{\varphi^{\frac{1}{\alpha-1}}\left(R_{U}-R_{f}\right)+R_{D}-R_{f}}, \\
\text { where } \varphi \equiv \frac{w(p)\left(R_{U}-R_{f}\right)}{w(1-p) \beta\left(R_{f}-R_{D}\right)} .
\end{gathered}
$$

Note that in absence of arbitrage $\varphi>0$ for all $0<p<1$ and that $\lambda_{U}^{*}$ is positive whenever $\varphi^{\frac{1}{1-\alpha}}>\frac{R_{U}-R_{f}}{R_{f}-R_{D}}$.

The stationary point $\lambda_{U}^{*}=\frac{\left(R_{f}-\frac{W_{0}}{W_{U}^{*}}\right)\left(-1-\varphi^{\frac{1}{\alpha-1}}\right)}{\varphi^{\frac{1}{\alpha-1}}\left(R_{U}-R_{f}\right)+R_{D}-R_{f}}$ is an unconstraint maximum if the second derivative of the objective function at this point is negative

$$
\begin{aligned}
V_{U G L}^{\prime \prime}\left(\lambda_{U}\right)= & \alpha(\alpha-1) w(p)\left(W_{U}^{*}\left(\lambda_{U}\left(R_{U}-R_{f}\right)+R_{f}\right)-W_{0}\right)^{\alpha-2} W_{U}^{2}\left(R_{U}-R_{f}\right)^{2} \\
& -\alpha(\alpha-1) w(1-p)(\beta)\left(W_{0}-W_{U}^{*}\left(\lambda_{U}\left(R_{D}-R_{f}\right)+R_{f}\right)\right)^{\alpha-2} \\
& W_{U}^{* 2}\left(R_{f}-R_{D}\right)^{2} .
\end{aligned}
$$

Note that $\alpha-1$ is smaller than zero. The stationary point $\lambda_{U}^{*}$ is a maximum if

$$
\begin{array}{r}
V_{U G L}^{\prime \prime}\left(\lambda_{U}^{*}\right)<0 \\
\varphi^{\frac{1}{1-\alpha}}<\frac{R_{U}-R_{f}}{R_{f}-R_{D}} .
\end{array}
$$


Note, that this is the condition for $\lambda_{U}^{*}=\frac{\left(R_{f}-\frac{W_{0}}{W_{U}^{*}}\right)\left(-1-\varphi^{\frac{1}{\alpha-1}}\right)}{\varphi^{\frac{1}{\alpha-1}}\left(R_{U}-R_{f}\right)+R_{D}-R_{f}}$ to be a maximum. However, as we shown above, for $\lambda_{U}^{*}$ to be positive we require $\varphi^{\frac{1}{1-\alpha}}>\frac{R_{U}-R_{f}}{R_{f}-R_{D}}$, implying, that $\lambda_{U}^{*}$ in fact is a minimum, given the requirement of non-negativity. Since the stationary point is unique this result implies that in the domain of positive $\lambda_{U}$ the value function for the case where a gain and a loss arise is convex.

The theorem of Weierstrass states that if the opportunity set is compact (i.e. closed and bounded) and nonempty and the objective function is continuous on the opportunity set, the objective has a global maximum either in the interior or on the boundary of the opportunity set. Thus, in our case the maximum is at one of the boundaries. So we can infer that in the case where the investor chooses to allocate his wealth in a way that there is a possible loss in the next period the optimal allocation is

$$
\lambda_{U}^{*}=\left\{\begin{array}{ll}
\frac{R_{f}-\frac{W_{0}}{W_{U}^{*}}}{R_{f}-R_{D}} & \text { if } V_{U G L}\left(\frac{R_{f}-\frac{W_{0}}{W_{U}^{*}}}{R_{f}-R_{D}}\right)>V_{U G L}(1) \text { and if } \frac{R_{f}-\frac{W_{0}}{W_{U}^{*}}}{R_{f}-R_{D}} \leq 1 \\
1 & \text { if } V_{U G L}(1)>V_{U G L}\left(\frac{R_{f}-\frac{W_{0}}{W_{U}^{*}}}{R_{f}-R_{D}}\right) \text { and if } \frac{R_{f}-\frac{W_{0}}{W_{U}^{*}}}{R_{f}-R_{D}} \leq 1
\end{array} .\right.
$$

\section{A.4 Optimal Asset Allocation in $\mathrm{t}=\mathrm{D}$ of the Investor who's Reference Point is his Initial Wealth}

\section{Proof of Proposition 4}

This proof follows the proof of proposition 1. Since the editing of the prospects implies different forms of the value function we have to treat the two possible cases separately. First consider the case where the investor chooses to allocate his wealth such that only gains are possible at the end of the second period. This case arises when

1. in $t=0$ it was optimal to choose $\lambda_{0}^{*}=1$ and if in $t=1$ the agent chooses $\frac{R_{f}-\frac{W_{0}}{W_{D}^{*}}}{R_{f}-R_{D}} \geq \lambda_{D} \geq 0$ and the parameters satisfy the relation $R_{U}>R_{f}>\frac{W_{0}}{W_{D}^{*}}>1$, when 
2. in $t=0$ it was optimal to choose $\lambda_{0}^{*}=\frac{R_{f}-1}{R_{f}-R_{D}}$ and if in $t=1$ the agent chooses $\frac{R_{f}-1}{R_{f}-R_{D}} \geq \lambda_{D} \geq 0$ or when

3. in $t=0$ it was optimal to choose $0 \leq \lambda_{0}^{*}<\frac{R_{f}-1}{R_{f}-R_{D}}$ and if in $t=1$ the agent chooses $\frac{R_{f}-\frac{W_{0}}{W_{D}^{*}}}{R_{f}-R_{D}} \geq \lambda_{D} \geq 0$ and the parameters satisfy the relation $R_{U}>R_{f}>\frac{W_{0}}{W_{D}^{*}}$.

Note that in the case where $\lambda_{D}$ equals the upper boundary neither a gain nor a loss occurs. However, in this section we treat this case as a zero gain.

The objective function writes

$$
\begin{aligned}
V_{D G G}\left(\lambda_{U}\right)= & v\left(W_{D D}-W_{0}\right)+w(p)\left(v\left(W_{D U}-W_{0}\right)-v\left(W_{D D}-W_{0}\right)\right) \\
= & (1-w(p))\left(W_{D}^{*}\left(\left(R_{D}-R_{f}\right) \lambda_{D}+R_{f}\right)-W_{0}\right)^{\alpha} \\
& +w(p)\left(W_{D}^{*}\left(\left(R_{U}-R_{f}\right) \lambda_{D}+R_{f}\right)-W_{0}\right)^{\alpha} .
\end{aligned}
$$

First consider the case where the constraints do not bind. The first and second derivative of the the objective function are

$$
\begin{array}{r}
V_{D G G}^{\prime}=\alpha w(p)\left(W_{D}^{*}\left(\left(R_{U}-R_{f}\right) \lambda_{D}+R_{f}\right)-W_{0}\right)^{\alpha-1} W_{D}^{*}\left(R_{U}-R_{f}\right) \\
+\alpha(1-w(p))\left(W_{D}^{*}\left(\lambda_{D}\left(R_{D}-R_{f}\right)+R_{f}\right)-W_{0}\right)^{\alpha-1} W_{D}^{*}\left(R_{D}-R_{f}\right) \\
V_{D G G}^{\prime \prime}=\left((1-w(p))\left(W_{D}^{*}\left(\lambda_{D}\left(R_{D}-R_{f}\right)+R_{f}\right)-W_{0}\right)^{\alpha-2}\left(R_{f}-R_{D}\right)^{2}\right. \\
\left.+w(p)\left(W_{D}^{*}\left(\left(R_{U}-R_{f}\right) \lambda_{D}+R_{f}\right)-W_{0}\right)^{\alpha-2}\left(R_{U}-R_{f}\right)^{2}\right) \alpha(\alpha-1) W_{D}^{* 2} .
\end{array}
$$

For $0 \leq w(p) \leq 1, R_{U}>R_{f} \geq 1>R_{D}, 0<\alpha<1$ and $W_{0}>0$ the second derivative of the objective function is negative, implying that the value function for the case where only gains arise is concave.

Solving the first order condition yields the optimal portfolio allocation for the unconstraint case

$$
\begin{gathered}
\lambda_{D}^{*}=\frac{\left(R_{f}-\frac{W_{0}}{W_{D}^{*}}\right)\left(1-\kappa^{\frac{1}{\alpha-1}}\right)}{\left(R_{U}-R_{f}\right) \kappa^{\frac{1}{\alpha-1}}+R_{f}-R_{D}}, \\
\text { where } \kappa \equiv \frac{w(p)\left(R_{U}-R_{f}\right)}{(1-w(p))\left(R_{f}-R_{D}\right)} .
\end{gathered}
$$


From the concavity of the objective function it follows that $\lambda_{D}^{*}$ is a maximum.

Next consider the case where the lower boundary, i.e. $\lambda_{D}=0$, is binding. Using the concavity property it follows that this cases arises whenever the unconstraint $\lambda_{D}^{*}$ is negative or equivalently $w(p) \leq \frac{R_{f}-R_{D}}{R_{U}-R_{D}}$.

If the upper boundary is binding then $\lambda_{D}^{*}=\frac{R_{f}-\frac{W_{0}}{W_{D}^{*}}}{R_{f}-R_{D}}$. In the case where $\frac{R_{f}-\frac{W_{0}}{W_{D}^{*}}}{R_{f}-R_{D}} \geq 1$ the optimal allocation is bounded by one, i.e. $\lambda_{D}^{*}=1$, when ever $\kappa^{\frac{1}{\alpha-1}} \leq \frac{R_{D}-\frac{W_{0}}{W_{D}^{*}}}{R_{U}-\frac{W_{0}}{W_{D}^{*}}}$. Note that this case can only arise if $0 \leq \lambda_{0}^{*}<\frac{R_{f}-1}{R_{f}-R_{D}}$, since we require $\frac{W_{0}}{W_{D}^{*}}<R_{D}$.

The second case, i.e. the one without segregation, arises when the agent chooses to allocate his wealth in a way such that there is a possible loss at the end of the next period.

This case arises when

1. in $t=0$ it was optimal to choose $\lambda_{0}^{*}=1$ and if in $t=1$ the agent chooses $1 \geq \lambda_{D} \geq \frac{\frac{W_{0}}{W_{D}^{*}}-R_{f}}{R_{U}-R_{f}}$ and the parameters satisfy the following relation $R_{U}>\frac{W_{0}}{W_{D}^{*}}>R_{f}>1$, when

2. in $t=0$ it was optimal to choose $\lambda_{0}^{*}=1$ and and if in $t=1$ the agent chooses $1 \geq \lambda_{D} \geq \frac{R_{f}-\frac{W_{0}}{W_{D}^{*}}}{R_{f}-R_{D}}$ and the parameters satisfy the following relation $R_{U}>R_{f}>\frac{W_{0}}{W_{D}^{*}}>1$, when

3. in $t=0$ it was optimal to choose $\lambda_{0}^{*}=\frac{R_{f}-1}{R_{f}-R_{D}}$ and and if in $t=1$ the agent chooses $\frac{R_{f}-1}{R_{f}-R_{D}} \leq \lambda_{D} \leq 1$ or when

4. in $t=0$ it was optimal to choose $0 \leq \lambda_{0}^{*} \leq \frac{R_{f}-1}{R_{f}-R_{D}}$ and and if in $t=1$ the agent chooses $1 \geq \lambda_{D} \geq \frac{R_{f}-\frac{W_{0}}{W_{D}^{*}}}{R_{f}-R_{D}}$ and the parameters satisfy the following relation $R_{U}>R_{f} \geq 1>\frac{W_{0}}{W_{D}^{*}}>R_{D}$.

The objective function writes

$$
\begin{aligned}
V_{D G L}\left(\lambda_{D}\right)= & w(p)\left(W_{D}^{*}\left(\left(R_{U}-R_{f}\right) \lambda_{D}+R_{f}\right)-W_{0}\right)^{\alpha} \\
& +w(1-p)(-\beta)\left(W_{0}-W_{D}^{*}\left(\left(R_{D}-R_{f}\right) \lambda_{D}+R_{f}\right)\right)^{\alpha} .
\end{aligned}
$$


First consider the case where the constraints do not bind. The first order condition writes

$$
\begin{gathered}
\quad \alpha w(1-p) \beta\left(W_{0}-W_{U}^{*}\left(\lambda_{D}\left(R_{D}-R_{f}\right)+R_{f}\right)\right)^{\alpha-1} W_{D}^{*}\left(R_{D}-R_{f}\right) \\
\left.+\alpha w(p)\left(W_{D}^{*}\left(\lambda_{D}\left(R_{U}-R_{f}\right)+R_{f}\right)-W_{0}\right)\right)^{\alpha-1} W_{D}^{*}\left(R_{U}-R_{f}\right)=0 .
\end{gathered}
$$

Solving the first order condition yields the stationary point for the optimal portfolio allocation for the unconstraint case

$$
\begin{gathered}
\lambda_{D}^{*}=\frac{\left(R_{f}-\frac{W_{0}}{W_{D}^{*}}\right)\left(-1-\varphi^{\frac{1}{\alpha-1}}\right)}{\varphi^{\frac{1}{\alpha-1}}\left(R_{U}-R_{f}\right)+R_{D}-R_{f}}, \\
\text { where } \varphi \equiv \frac{w(p)\left(R_{U}-R_{f}\right)}{w(1-p) \beta\left(R_{f}-R_{D}\right)} .
\end{gathered}
$$

Note that in absence of arbitrage $\varphi>0$ for all $0<p<1$ and that the stationary point $\lambda_{D}^{*}=\frac{\left(R_{f}-\frac{W_{0}}{W_{D}^{*}}\right)\left(-1-\varphi^{\frac{1}{\alpha-1}}\right)}{\varphi^{\frac{1}{\alpha-1}}\left(R_{U}-R_{f}\right)+R_{D}-R_{f}}$ is positive

1. in the case where $\lambda_{0}^{*}=1$ and $1 \geq \lambda_{D} \geq \frac{\frac{W_{0}}{W_{D}^{*}}-R_{f}}{R_{U}-R_{f}}$ and $R_{U}>\frac{W_{0}}{W_{D}^{*}}>R_{f}>1$ if $\varphi^{\frac{1}{1-\alpha}}<\frac{R_{U}-R_{f}}{R_{f}-R_{D}}$

2. in the case where $\lambda_{0}^{*}=1$ and $1 \geq \lambda_{D} \geq \frac{R_{f}-\frac{W_{0}}{W_{D}^{*}}}{R_{f}-R_{D}}$ and $R_{U}>R_{f}>\frac{W_{0}}{W_{D}^{*}}>1$ if $\varphi^{\frac{1}{1-\alpha}}>\frac{R_{U}-R_{f}}{R_{f}-R_{D}}$

3. in the case where $\lambda_{0}^{*}=\frac{R_{f}-1}{R_{f}-R_{D}}$ and $\frac{R_{f}-1}{R_{f}-R_{D}} \leq \lambda_{D} \leq 1$ if $\varphi^{\frac{1}{1-\alpha}}>\frac{R_{U}-R_{f}}{R_{f}-R_{D}}$ and

4. in the case where $0 \leq \lambda_{0}^{*} \leq \frac{R_{f}-1}{R_{f}-R_{D}}$ and $1 \geq \lambda_{D} \geq \frac{R_{f}-\frac{W_{0}}{W_{D}^{*}}}{R_{f}-R_{D}}$ and $R_{U}>$ $R_{f} \geq 1>\frac{W_{0}}{W_{D}^{*}}>R_{D}$ if $\varphi^{\frac{1}{1-\alpha}}>\frac{R_{U}-R_{f}}{R_{f}-R_{D}}$.

Together with the following condition about the second derivative of the objective function we can draw conclusions on the curvature of $V_{D G L}$ for positive $\lambda_{D}$ 


$$
\begin{array}{r}
V_{D G L}^{\prime \prime}=\left(w(p)\left(W_{D}^{*}\left(\left(R_{U}-R_{f}\right) \lambda_{D}+R_{f}\right)-W_{0}\right)^{\alpha-2}\left(R_{U}-R_{f}\right)^{2}\right. \\
\left.-w(1-p) \beta\left(W_{0}-W_{D}^{*}\left(\left(R_{D}-R_{f}\right) \lambda_{D}+R_{f}\right)\right)^{\alpha-2}\left(R_{f}-R_{D}\right)^{2}\right) \alpha(\alpha-1) W_{D}^{2}
\end{array}
$$

The expression is smaller that zero whenever the term in the brackets is positive or equivalently whenever $\varphi^{\frac{1}{1-\alpha}}<\frac{R_{U}-R_{f}}{R_{f}-R_{D}}$. From this we deduce the following conclusions.

1. In the case where $\lambda_{0}^{*}=1$ and $1 \geq \lambda_{D} \geq \frac{\frac{W_{0}}{W_{D}^{*}}-R_{f}}{R_{U}-R_{f}}$ and $R_{U}>\frac{W_{0}}{W_{D}^{*}}>$ $R_{f}>1$ the objective function is concave and thus the stationary point $\frac{\left(R_{f}-\frac{W_{0}}{W_{D}^{*}}\right)\left(-1-\varphi^{\frac{1}{\alpha-1}}\right)}{\varphi^{\frac{1}{\alpha-1}}\left(R_{U}-R_{f}\right)+R_{D}-R_{f}}$ is a maximum.

2. In the case where $\lambda_{0}^{*}=1$ and $1 \geq \lambda_{D} \geq \frac{R_{f}-\frac{W_{0}}{W_{D}^{*}}}{R_{f}-R_{D}}$ and $R_{U}>R_{f}>$ $\frac{W_{0}}{W_{D}^{*}}>1$ the objective function is convex and thus the stationary point $\frac{\left(R_{f}-\frac{W_{0}}{W_{D}^{*}}\right)\left(-1-\varphi^{\frac{1}{\alpha-1}}\right)}{\varphi^{\frac{1}{\alpha-1}}\left(R_{U}-R_{f}\right)+R_{D}-R_{f}}$ is a minimum, so that the maximum is at one of the boundaries.

3. In the case where $\lambda_{0}^{*}=\frac{R_{f}-1}{R_{f}-R_{D}}$ and $\frac{R_{f}-1}{R_{f}-R_{D}} \leq \lambda_{D} \leq 1$ the objective function is convex and thus the stationary point $\frac{\left(R_{f}-\frac{W_{0}}{W_{D}^{*}}\right)\left(-1-\varphi^{\frac{1}{\alpha-1}}\right)}{\varphi^{\frac{1}{\alpha-1}}\left(R_{U}-R_{f}\right)+R_{D}-R_{f}}$ is a minimum, so that the maximum is at one of the boundaries.

4. In the case where $0 \leq \lambda_{0}^{*} \leq \frac{R_{f}-1}{R_{f}-R_{D}}$ and $1 \geq \lambda_{D} \geq \frac{R_{f}-\frac{W_{0}}{W_{D}^{*}}}{R_{f}-R_{D}}$ and $R_{U}>R_{f} \geq 1>\frac{W_{0}}{W_{D}^{*}}>R_{D}$ the objective function is convex and thus the stationary point $\frac{\left(R_{f}-\frac{W_{0}}{W_{D}^{*}}\right)\left(-1-\varphi^{\frac{1}{\alpha-1}}\right)}{\varphi^{\frac{1}{\alpha-1}}\left(R_{U}-R_{f}\right)+R_{D}-R_{f}}$ is a minimum, so that the maximum is at one of the boundaries. 
In the case where $\lambda_{0}^{*}=1$ and $R_{U}>\frac{W_{0}}{W_{D}^{*}}>R_{f}>1$ the optimal asset allocation is in the unconstrained case

$$
\begin{gathered}
\lambda_{D}^{*}=\frac{\left(R_{f}-\frac{W_{0}}{W_{D}^{*}}\right)\left(-1-\varphi^{\frac{1}{\alpha-1}}\right)}{\varphi^{\frac{1}{\alpha-1}}\left(R_{U}-R_{f}\right)+R_{D}-R_{f}}, \\
\text { where } \varphi \equiv \frac{w(p)\left(R_{U}-R_{f}\right)}{w(1-p) \beta\left(R_{f}-R_{D}\right)} .
\end{gathered}
$$

From the concavity of the objective function we know that $\lambda_{D}^{*}=1$ whenever $\frac{\left(R_{f}-\frac{W_{0}}{W_{D}^{*}}\right)\left(-1-\varphi^{\frac{1}{\alpha-1}}\right)}{\varphi^{\frac{1}{\alpha-1}}\left(R_{U}-R_{f}\right)+R_{D}-R_{f}} \geq 1$ or equivalently $\varphi^{\frac{1}{\alpha-1}} \leq \frac{\frac{W_{0}}{W_{D}^{*}}-R_{D}}{R_{U}-\frac{W_{0}}{W_{D}^{*}}}$. Note that this requirement is consistent with the requirement of positivity, i.e. $\varphi^{\frac{1}{1-\alpha}}<$ $\frac{R_{U}-R_{f}}{R_{f}-R_{D}}$. Further we know that $\lambda_{D}^{*}=\frac{\frac{W_{0}}{W_{D}^{*}}-R_{f}}{R_{u}-R_{f}}$ whenever $\frac{\left(R_{f}-\frac{W_{0}}{W_{D}^{*}}\right)\left(-1-\varphi^{\frac{1}{\alpha-1}}\right)}{\varphi^{\frac{1}{\alpha-1}}\left(R_{U}-R_{f}\right)+R_{D}-R_{f}} \leq$ $\frac{\frac{W_{0}}{W_{D}^{*}}-R_{f}}{R_{u}-R_{f}}$. This arises in the special case where $\frac{W_{0}}{W_{D}^{*}}=R_{f}$ and implies that $\lambda_{D}^{*}=0$. is

In the case where $\lambda_{0}^{*}=1$ and $R_{U}>R_{f}>\frac{W_{0}}{W_{D}^{*}}>1$ the optimal allocation

$$
\lambda_{D G L}^{*}=\left\{\begin{array}{ll}
\frac{R_{f}-\frac{W_{0}}{W_{D}^{*}}}{R_{f}-R_{D}} & \text { if } V_{G L}\left(\frac{R_{f}-\frac{W_{0}}{W_{D}^{*}}}{R_{f}-R_{D}}\right)>V_{G L}(1) \\
1 & \text { if } V_{D G L}(1)>V_{G L}\left(\frac{R_{f}-\frac{W_{0}}{W_{D}^{*}}}{R_{f}-R_{D}}\right)
\end{array} .\right.
$$

In the case where $\lambda_{0}^{*}=\frac{R_{f}-1}{R_{f}-R_{D}}$ the optimal allocation is

$$
\lambda_{D G L}^{*}=\left\{\begin{array}{ll}
\frac{R_{f}-1}{R_{f}-R_{D}} & \text { if } V_{G L}\left(\frac{R_{f}-1}{R_{f}-R_{D}}\right)>V_{G L}(1) \\
1 & \text { if } V_{G L}(1)>V_{G L}\left(\frac{R_{f}-1}{R_{f}-R_{D}}\right)
\end{array} .\right.
$$

In the case where $0 \leq \lambda_{0}^{*} \leq \frac{R_{f}-1}{R_{f}-R_{D}}$ and and $R_{U}>R_{f} \geq 1>\frac{W_{0}}{W_{D}^{*}}>R_{D}$

$$
\lambda_{D G L}^{*}=\left\{\begin{array}{ll}
\frac{R_{f}-\frac{W_{0}}{W_{D}^{*}}}{R_{f}-R_{D}} & \text { if } V_{G L}\left(\frac{R_{f}-\frac{W_{0}}{W_{D}^{*}}}{R_{f}-R_{D}}\right)>V_{G L}(1) \\
1 & \text { if } V_{G L}(1)>V_{G L}\left(\frac{R_{f}-\frac{W_{0}}{W_{D}^{*}}}{R_{f}-R_{D}}\right)
\end{array} .\right.
$$

The third case arises when the agent chooses to allocate his wealth in $t=1$ in a way that in $t=2$ he makes two losses. This happens after a first period choice of $\lambda_{0}^{*}=1$ and if the investor chooses a $0 \leq \lambda_{D}^{*} \leq \frac{\frac{W_{0}}{W_{D}^{*}}-R_{f}}{R_{U}-R_{f}}$. 
The objective function writes

$$
\begin{aligned}
& V_{D L L}=-w(1-p)(\beta)\left(W_{0}-W_{D}^{*}\left(\left(R_{D}-R_{f}\right) \lambda_{D}+R_{f}\right)\right)^{\alpha} \\
& \quad-(1-w(1-p))(\beta)\left(W_{0}-W_{D}^{*}\left(\left(R_{U}-R_{f}\right) \lambda_{D}+R_{f}\right)\right)^{\alpha} .
\end{aligned}
$$

First consider the case where the constraints do not bind. The first and second derivative of the the objective function are

$$
\begin{array}{r}
V_{D L L}^{\prime}=-\alpha w(1-p) \beta\left(W_{0}-W_{D}^{*}\left(\left(R_{D}-R_{f}\right) \lambda_{D}+R_{f}\right)\right)^{\alpha-1}(-1) W_{D}^{*}\left(R_{D}-R_{f}\right) \\
-\alpha(1-w(1-p)) \beta\left(W_{0}-W_{D}^{*}\left(\left(R_{U}-R_{f}\right) \lambda_{D}+R_{f}\right)\right)^{\alpha-1}(-1) W_{D}^{*}\left(R_{U}-R_{f}\right) \\
V_{D L L}^{\prime \prime}=\alpha(1-\alpha) w(1-p) \beta\left(W_{0}-W_{D}^{*}\left(\left(R_{D}-R_{f}\right) \lambda_{D}+R_{f}\right)\right)^{\alpha-2} W_{D}^{* 2}\left(R_{D}-R_{f}\right)^{2} \\
+\alpha(1-\alpha)(1-w(1-p)) \beta\left(W_{0}-W_{D}^{*}\left(\left(R_{U}-R_{f}\right) \lambda_{D}+R_{f}\right)\right)^{\alpha-2} W_{D}^{* 2}\left(R_{U}-R_{f}\right)^{2} .
\end{array}
$$

For $0 \leq w(p) \leq 1, R_{U}>R_{f} \geq 1>R_{D}, 0<\alpha<1$ and $W_{0}>0$ the second derivative of the objective function is positive, implying that the value function for the case where only losses arise is convex. It follows that in our maximization problem the boundaries are the stationary points.

The stationary points are zero and $\frac{\frac{W_{0}}{W_{D}^{*}-R_{f}}}{R_{U}-R_{f}}$, if $\frac{\frac{W_{0}}{W_{D}^{*}}-R_{f}}{R_{U}-R_{f}}<1$, or zero and one in the other case. So that in the case where $\lambda_{0}^{*}=1$ and $0 \leq \lambda_{D}^{*} \leq \frac{\frac{W_{0}}{W_{D}^{*}}-R_{f}}{R_{U}-R_{f}}$ the optimal allocation is in the case where $\frac{W_{0}}{W_{D}^{*}}<R_{U}$ the optimal asset allocation is

$$
\lambda_{D L L}^{*}= \begin{cases}0 & \text { if } V_{L L}(0)>V_{L L}\left(\frac{\frac{W_{0}}{W_{D}^{*}}-R_{f}}{R_{U}-R_{f}}\right) \\ \frac{W_{0}}{W_{D}^{*}-R_{f}} & \text { if } V_{L L}\left(\frac{\frac{W_{0}}{W_{D}^{*}}-R_{f}}{R_{U}-R_{f}}\right)>V_{L L}(0)\end{cases}
$$

and in the case where $\frac{W_{0}}{W_{D}^{*}} \geq R_{U}$

$$
\lambda_{D L L}^{*}=\left\{\begin{array}{ll}
0 & \text { if } V_{L L}(0)>V_{L L}(1) \\
1 & \text { if } V_{L L}(1)>V_{L L}(0)
\end{array} .\right.
$$




\section{References}

[1] Barberis, Nicholas, Ming Huang And Tano Santos (2001): "Prospect Theory and Asset Prices", Quarterly Journal of Economics, $116,1-53$.

[2] Benartzi, Shlomo And Richard Thaler (1995): "Myopic loss aversion and the equity premium puzzle", Quarterly Journal of Economics, 110, 73-92.

[3] De Giorgi, Enrico, Thorsten Hens and Haim Levy (2004): "Existence of CAPM Equilibria with ProspectTheory Preferences", $\mathrm{Na}$ tional Centre of Competence in Research Financial Valuation and Risk Management Working Paper No. $85,1-42$.

[4] Kahneman, Daniel and Amos Tversky (1979): "Prospect Theory: An Analysis of Decision under Risk", Econometrica, 47, 263-291.

[5] Odean, Terrance (1998A): "Are Investors Reluctant to Realize their Losses", Journal of Finance, 53, 1775-1798.

[6] Shefrin, Hersh and Meir Statman (1985): "The Disposition to Sell Winners too Early and Ride Losers too Long: Theory and Evidence", Journal of Finance, 40, 777-790.

[7] Thaler, Richard H. And Eric J. Johnson (1990): "Gambling with the House Money and Trying to Break Even: The Effects of Prior Outcomes on Risky Choice", Management Science, 643-660.

[8] Tversky, Amos And Daniel Kahneman (1992): "Advances in prospect theory: cumulative representation of uncertainty", Journal of Risk and Uncertainty, 5, 297-323.

[9] Weber, Martin and Colin F. Camerer (1998): "The Disposition Effect in Securities Trading: An Experimental Analysis", Journal of Economic Behavior \& Organization, 33, 167-184.

[10] Kyle, Albert S., Hui Ou-Yang, And Wei Xiong 